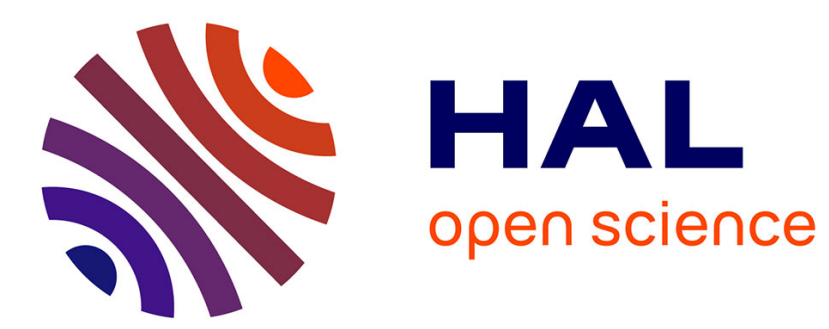

\title{
An experimental study of dipolar vortex structures in a stratified fluid
}

J. Flór, J. van Heijst

\section{To cite this version:}

J. Flór, J. van Heijst. An experimental study of dipolar vortex structures in a stratified fluid. Journal of Fluid Mechanics, 1994, 279 (1), pp.101-133. 10.1017/S0022112094003836 . hal-02140422

\section{HAL Id: hal-02140422 \\ https://hal.science/hal-02140422}

Submitted on 23 Sep 2020

HAL is a multi-disciplinary open access archive for the deposit and dissemination of scientific research documents, whether they are published or not. The documents may come from teaching and research institutions in France or abroad, or from public or private research centers.
L'archive ouverte pluridisciplinaire HAL, est destinée au dépôt et à la diffusion de documents scientifiques de niveau recherche, publiés ou non, émanant des établissements d'enseignement et de recherche français ou étrangers, des laboratoires publics ou privés. 


\title{
An experimental study of dipolar vortex structures in a stratified fluid
}

\author{
By J. B. FLÓR AND G. J. F. VAN HEIJST \\ J. M. Burgers Centre for Fluid Mechanics, Department of Technical Physics, Eindhoven \\ University of Technology, P- Box 513,5600MB Eindhoven, The Netherlands
}

This paper describes laboratory experiments on dipolar vortex structures in a linearly stratified fluid. The dipoles are generated by a pulsed horizontal injection of a small volume of fluid, by which a localized three-dimensionally turbulent flow region is created. After the subsequent gravitational collapse the flow becomes approximately two-dimensional, and eventually a single vortex dipole emerges, as the result of the selforganizing properties of such flows. The flow evolution has been visualized both by dye and tracer particles, through which qualitative as well as quantitative information was obtained. By application of digital image analysis, the spatial distribution of the velocity $\boldsymbol{v}$, vorticity $\omega$ and stream function $\psi$ were determined. It was found that dipoles in the turbulent-injection experiments are characterized by a nonlinear sinh-like relationship between $\omega$ and $\psi$, whereas in the case of laminar injection the $(\omega, \psi)$-scatter plots of the dipoles reveal a linear relationship. Notwithstanding these differences, both types of dipoles show a dynamical structure that agrees very well with the Lamb-Chaplygin dipole, as was found by comparing the size, position of maximum vorticity, cross-sectional distributions of $v$ and $\omega$, characteristics of the $(\omega, \psi)$ relationship, and the translation speed of the experimental and the model dipole.

\section{Introduction}

It is now well-known that vortices are characteristic features in the large-scale geophysical flow systems: in particular from satellite imagery it became clear that both the Earth's atmosphere and its oceans contain a wealth of horizontal structures on a range of spatial scales. The most intriguing ones are the large-scale vortices which generally possess a long lifetime. A common feature of these vortices is that to a good approximation their flow field is two-dimensional. In geophysical situations this twodimensionality is mainly caused by the planetary background rotation, but is partially also due to the density stratification (in particular relevant in the ocean) and to the twodimensional nature of the flow domain itself (the atmosphere and the oceans are relatively thin shells). However, each of these mechanisms can individually cause the flow to become two-dimensional, as has been demonstrated by a variety of experiments in rotating and stratified fluids as well as in soap films. In addition, magnetic forces may influence the flow in a dynamically equivalent way, and similar two-dimensional flow features have indeed been observed in a number of magneto-hydrodynamic situations. In this respect, the dynamics of two-dimensional flows is also relevant to the dynamics of tokamak-confined plasmas, and to certain astrophysical situations.

The most common two-dimensional vortex type is the single monopolar vortex which usually has a circular or elliptical shape. The next type is the dipolar vortex, consisting of two closely packed patches of oppositely signed vorticity. In addition, 
numerical and experimental studies have revealed the existence of a tripolar vortex, which may emerge as the end product of an initially unstable isolated monopolar vortex. The monopolar and tripolar vortex both contain net angular momentum, as evident from the rotational motion of the structure as a whole; in the absence of any background advective flow field or $\beta$-effect (due to the planet's spherical shape), these vortices are fixed in space. In contrast, the dipolar vortex possesses a self-propelling mechanism associated with its particular vorticity distribution: the symmetric dipole translates along a straight line (the trajectory is curved when the dipole is asymmetric). In other words, the dipolar vortex structure contains net linear momentum.

Partially because of their relevance to geophysical flow systems, the dynamics of two-dimensional vortices has been studied during the last two decades by an increasing number of investigators. In a large number of papers, the dynamics of quasi-twodimensional vortices (including stability, decay, interaction properties, effects of background flow or vorticity gradients) has been analysed theoretically or by numerical simulations. Along with these studies, laboratory experiments have been performed on vortices in rotating fluids, stratified fluids, magneto-hydrodynamic flows and even in soap films. The vortices in these different experimental situations shows certain similarities. For a recent review on vortices in a rotating fluid, the reader is referred to Hopfinger \& van Heijst (1993).

In non-rotating continuously stratified fluids, vortex structures usually have a characteristic pancake-like shape (see Lin \& Pao 1979) in the sense that their vertical dimensions are small compared to their horizontal size. The formation of dipolar vortex structures, as well as their dynamical properties, in a stratified fluid system have been investigated by Voropayev \& Filippov (1985) and Voropayev, Afanasyev \& Filippov (1991), and independently by van Heijst \& Flór $(1989 a, b)$. In these experiments, the dipoles were created by horizontal injection of fluid, i.e. by the introduction of some horizontal impulse. Also, Caperan \& Verron (1988) have performed experiments on vortices in a stratified fluid, in particular on the merging of like-signed monopoles and the interaction of oppositely signed monopoles leading to the formation of a dipolar vortex. Nguyen Duc \& Sommeria (1988) have carried out experiments on dipolar vortices in a thin layer of mercury subject to a uniform magnetic field, and these vortices were found to have the same global characteristics as their counterparts observed in rotating or stratified fluids. As mentioned earlier, the two-dimensionality of the flow geometry can also lead to the formation of twodimensional vortex structures. This was nicely demonstrated by the soap-film experiments of Couder \& Basdevant (1986) in which dipolar vortices were observed in the wake of a thin cylinder that was towed through the film.

The present paper describes experiments on dipolar vortex structures in a linearly stratified fluid, which were produced by pulsed horizontal injection of a small amount of fluid of matching density. The formation process and the dipole structure has been described to some extent in earlier papers by Voropayev \& Filippov (1985) and Voropayev et al. (1991) and by the present authors (van Heijst \& Flór 1989a,b). In their experiments, Voropayev and co-workers mainly considered dipoles created by laminar injection, and they studied the dipolar structure in terms of the point-force action (momentum) of the fluid injection. Also, they gave substantial attention to the entrainment of ambient fluid into the expanding dipole, for which they developed a theoretical model. The present study, however, is aimed at measuring precisely the dynamical structure of the quasi-stationary vortex dipole. For that purpose a digital image-analysis technique was used, which allowed very accurate determination of the spatial distribution of the flow field and its evolution. In this way quantitative 
information could be obtained about the horizontal velocity field and the associated distribution of the vertical vorticity $\omega$ and the stream function $\psi$. Moreover, by appropriate correction for the dipole's translation, the relation between $\omega$ and $\psi$ could be determined from so-called scatter plots. In addition, the evolution of the vortex dipole during its viscous decay has been considered, although the main results of that work will be published in a separate paper.

The present paper is organized as follows: the experimental arrangement is described in $\S 2$, which is followed by a description of the qualitative observations in $\S 3$. Some theoretical considerations about the vortex dipole, including a concise description of the Lamb-Chaplygin model and some measurable properties that can be derived from it, are presented in $\S 4$. Next, $\S 5$ describes the quantitative measurements of the flow characteristics, i.e. the distributions of velocity, vorticity and stream function, the $(\omega, \psi)$-scatter plot and the translation speed of the dipole. The observational data are compared with those predicted by the Lamb-Chaplygin model. Finally, a discussion of the results and some conclusions are given in $\S 6$.

\section{Experimental arrangement}

The experiments reported in this paper were performed in a $1 \mathrm{~m} \times 1 \mathrm{~m}$ Perspex tank with a working depth of $0.3 \mathrm{~m}$. The tank was filled with a linearly stratified salt solution, by applying the well-known two-tank method (Fortuin 1960). An isolated turbulent region was created (at $t=0 \mathrm{~s}$ ) by injecting a small amount of fluid (typically a few $\mathrm{ml}$ ) horizontally through a thin nozzle during a short period of time $\delta t$ (typically $1 \mathrm{~s})$ at a constant rate $Q\left(\mathrm{ml} \mathrm{s}^{-1}\right)$. Care was taken that the density of the injected fluid exactly matched that of the ambient fluid at that level. The injection nozzle was positioned at exactly half-depth; the total water depth in the tank was varied in the range from 16 to $20 \mathrm{~cm}$. The injection parameters were controlled by using a traversing mechanism in which one or more syringes with fluid could be fitted; in this way a prescribed volume could be injected at a prescribed speed. For the majority of the experiments the Reynolds number $R e=U d / \nu$ based on the nozzle diameter $d$ $(=2 \mathrm{~mm})$ and the injection velocity $U$, with $v$ being the kinematic viscosity of the fluid, was larger than 2000, thus ensuring a fully developed turbulent jet flow. Experiments were performed with different stratifications with the Brunt-Väisälä frequency $N$ ranging from $1.2 \mathrm{rad} \mathrm{s}^{-1}$ to $3 \mathrm{rad} \mathrm{s}^{-1}$. The evolving flow was visualized by adding fluorescein to the injected fluid, which was illuminated by a slit light from the side; the evolution of the dye pattern was recorded photographically by taking pictures from above with a remote-controlled photo camera that was mounted at some distance (approximately $2 \mathrm{~m}$ ) above the frec surface. In some cases a mirror was placed along one of the tank walls at an angle of approximately $45^{\circ}$ with the horizontal, so that the evolving dye patch could be monitored simultaneously both from above and from the side by a single camera. This technique provided quantitative information about the thickness and the horizontal dimensions of the turbulent patch. In order to measure the characteristics of the horizontal velocity field of the evolving flow, small $(1 \mathrm{~mm}$ diameter) polystyrene tracer particles with a density of $1.034 \mathrm{~g} \mathrm{~cm}^{-3}$ were added to the fluid, such that they became evenly distributed in a horizontal layer of roughly $0.5 \mathrm{~cm}$ thickness. The nozzle was carefully positioned in order to ensure that the in jection took place exactly at this level; the planar flow arising after the gravitational collapse of the injected fluid region was recorded by taking streak photographs from above. In addition, shadowgraph visualization was used in order to obtain information about the 
(a)

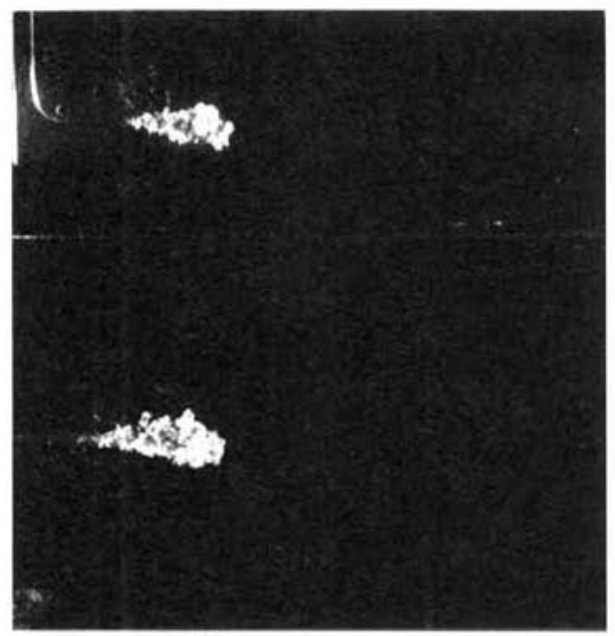

(c)

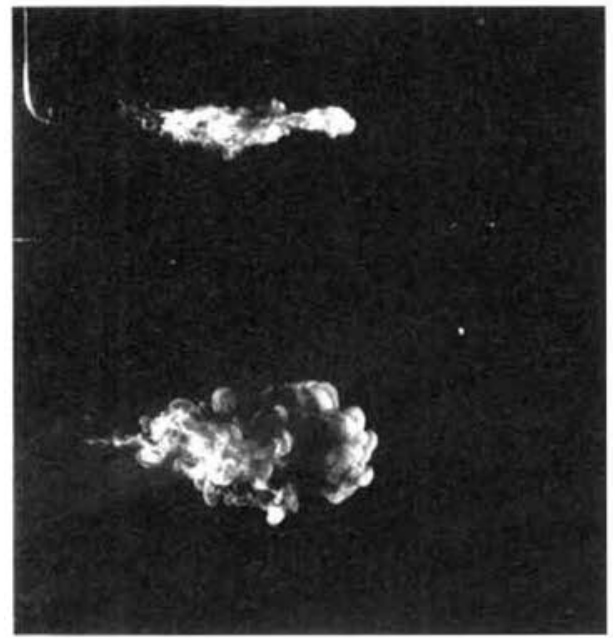

(e)

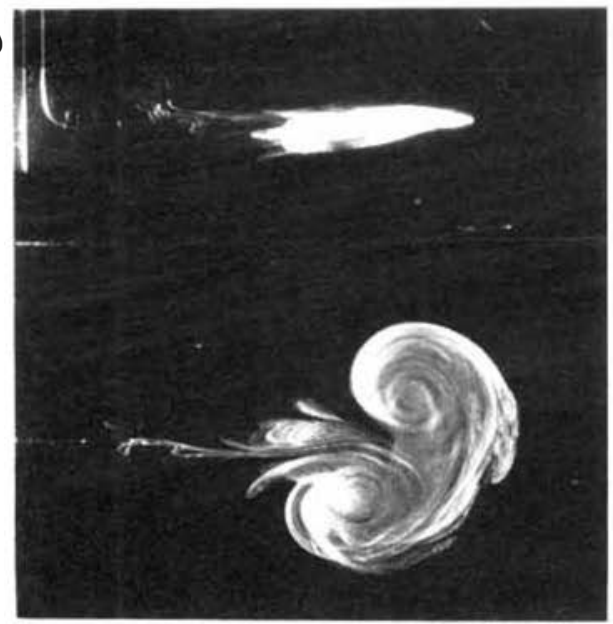

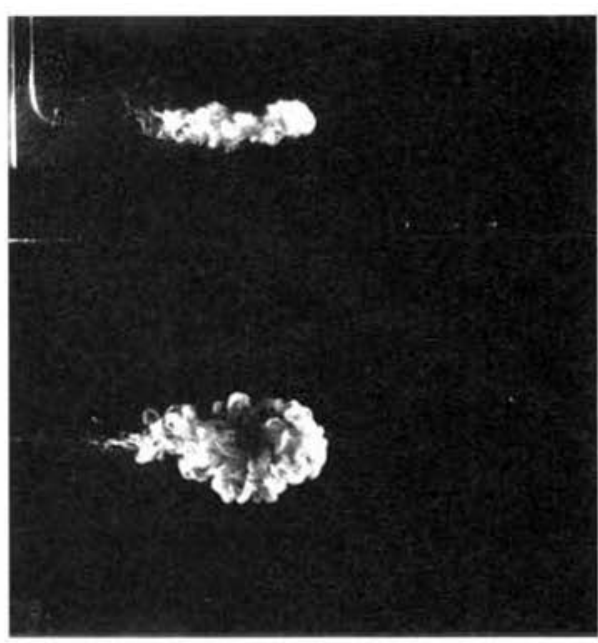

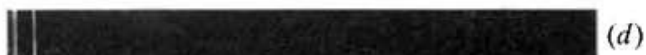

(d)
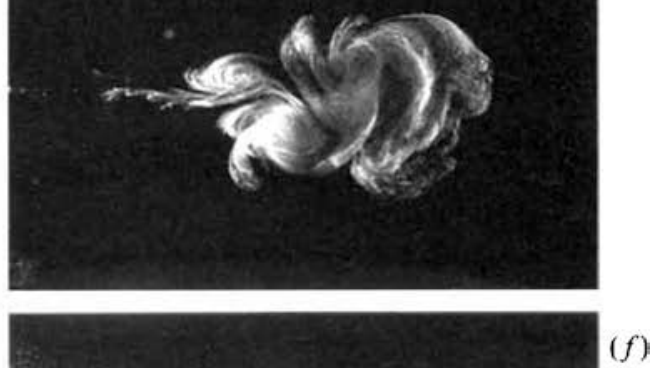

$(f)$

FIGURE 1. Sequence of photographs showing the evolution of an isolated turbulent region -created by a pulsed horizontal injection of fluid in a linearly stratified fluid - and the emergence of a dipolar vortex. Each photograph presents a top view and a side view (lower and upper part of the picture, respectively) of the dye-visualized flow: for this purpose a mirror was placed at an angle of $45^{\circ}$ along 
(turbulent) flow structure in the subsequent stages of the experiment as well as about the occurrence of internal waves.

\section{Qualitative observations}

The evolution of the turbulent patch and the subsequent formation of a dipolar vortex is most conveniently observed from dye visualizations of the flow, as presented in figure 1. The photographs were taken from above, with a mirror placed along one of the tank walls, thus enabling to obtain both a plan view and a side view of the dye structure on one picture: the upper and lower parts of each photograph show the side view and the plan view of the evolving dye structure, respectively. Initially, the injected flow is essentially three-dimensional, and the turbulent motion is confined in a coneshaped region (figure $1 a$ ). The turbulent region grows in vertical direction due to entrainment of ambient stratified fluid, until the kinetic energy of the turbulent eddies balances the potential energy required for the mixing of the entrained stratified fluid inside the patch. At that stage, the patch gradually collapses under gravity, as can be seen from the flattening of the dye structure (figure $1 b$ ): The mixed fluid starts to intrude into the quiescent ambient fluid. In the next stage the gravitationally driven intrusion stops, and a flattened region with mainly horizontal motions results (figure $1 c, d)$. It is clearly observed from the sequence of photographs (figure $1 b-d$ ) that the horizontal eddies show a gradual growth in size due to the merging of like-sign vortices - an effect of the inverse energy cascade present in two-dimensional flows - implying a spectral flux of kinetic energy from smaller to larger lengthscales. This merging of vortices eventually gives rise to the formation of a flat pancake-shaped dipolar vortex, see figure $1(e, f)$.

In some experiments it was observed that internal waves, caused by the gravitational collapse of the three-dimensional turbulent region, interact with the vortical flow region: dyed fluid is seen to be pushed radially outwards in a wave-like fashion from an apparent centre coinciding approximately with the centre of the dye patch. These internal waves are quickly damped, and are believed to play an insignificant role in the further evolution of the actual dipolar structure. A detailed study of the evolution of the turbulent patch resulting from the pulsed horizontal jet is given by Fernando, van Heijst \& Fonseka (1994).

Once the main characteristics of the dipolar vortex become distinguishable, see figure $1(d)$, the primary dipole vortex is observed to be surrounded by (two) secondary vortices. These secondary vortices are advected around the primary dipole, and subsequently partly left behind (remains are visible as the dye tails in figure $1 e, f$ ), partly entrained into the dipolar structure. At that stage the dipole formation is completed, and the dipolar vortex continues to translate quasi-steadily along a straight trajectory while preserving its shape.

The photograph in figure $1(f)$ clearly reveals the pancake-like shape of the visualized vortex region: the horizontal scale is much larger than the vertical lengthscale. In view of this shape one may define two different Reynolds numbers: a horizontal Reynolds number $R e_{h}=U_{0} D / \nu$, based on the translation velocity of the dipole $U_{0}$ and its diameter $D$, and a vertical Reynolds number $R e_{v}=2 \sigma U_{0} / \nu$, with $2 \sigma$ a measure of the dipole thickness. Soon after the dipole formation is completed these

one of the walls of the tank. The deviation between the frontal position of the dipole in the side view and that in the top view is due to the difference in optical distance relative to the photo camera. The photographs were taken at (a) $t=0.5 \mathrm{~s},(b) 3 \mathrm{~s},(c) 6 \mathrm{~s},(d) 35 \mathrm{~s},(e) 80 \mathrm{~s},(f) 400 \mathrm{~s}$. Experimental parameters: $Q=12 \mathrm{ml} \mathrm{s}^{-1}, \delta t=0.25 \mathrm{~s}$ and $N=1.2 \mathrm{rad} \mathrm{s}^{-1}$. 


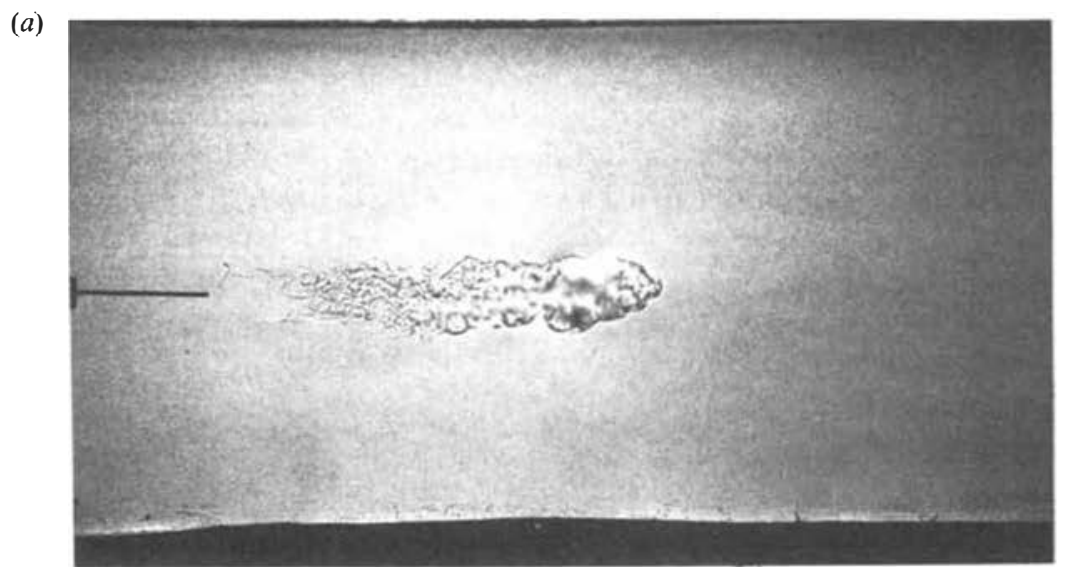

(b)

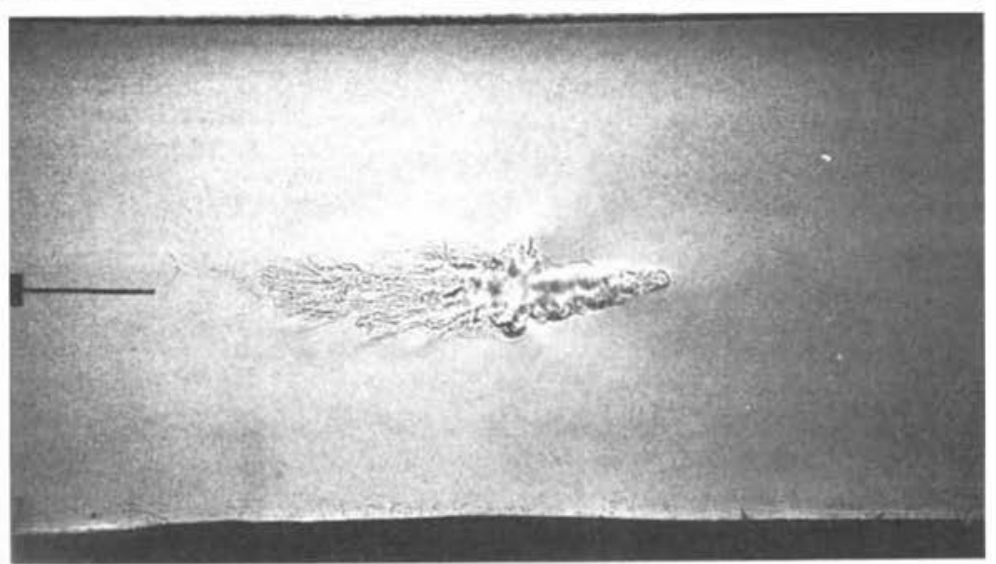

(c)

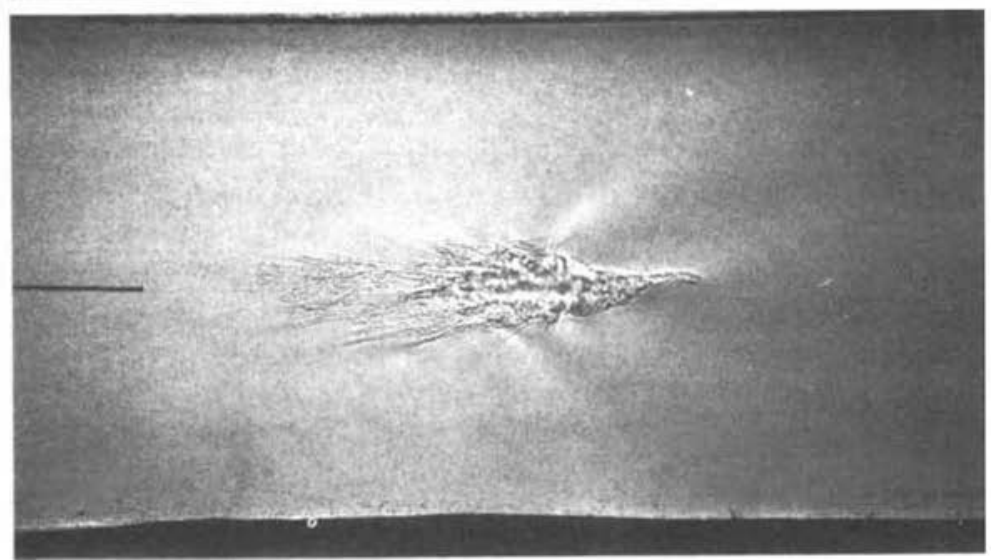

FIGURE $2(a-c)$. For caption see facing page.

Reynolds numbers have typical values $R e_{h}=1000$ and $R e_{v}=300$. During the course of an experiment these values decay rapidly to lower values of typically $O(100)$ and $O(10)$, respectively, indicating that vertical diffusion of vorticity is dominant.

Since the dipolar vortex evolves from an initially three-dimensional turbulent flow - in which the vorticity is likely to have an irregular distribution - its shape is not always perfectly symmetric: depending on the net vorticity contained in the vortices left behind in its wake, the dipole may be asymmetric and thus move along a curved 
$(d)$

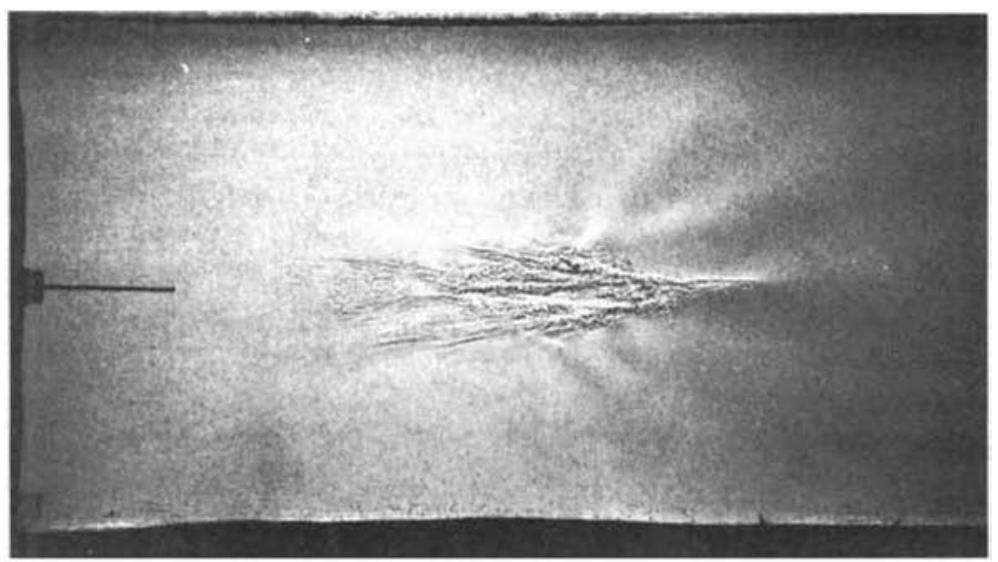

(e)

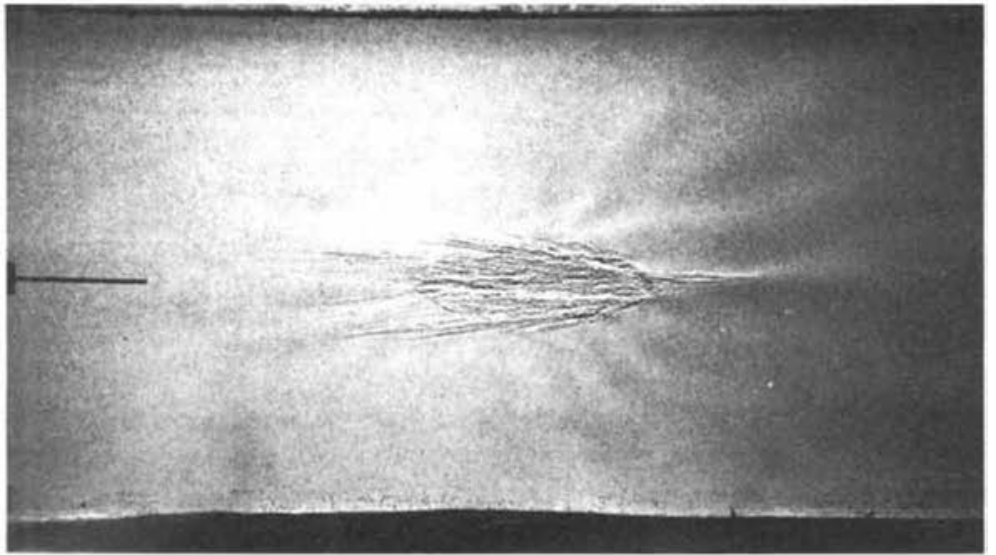

$(f)$

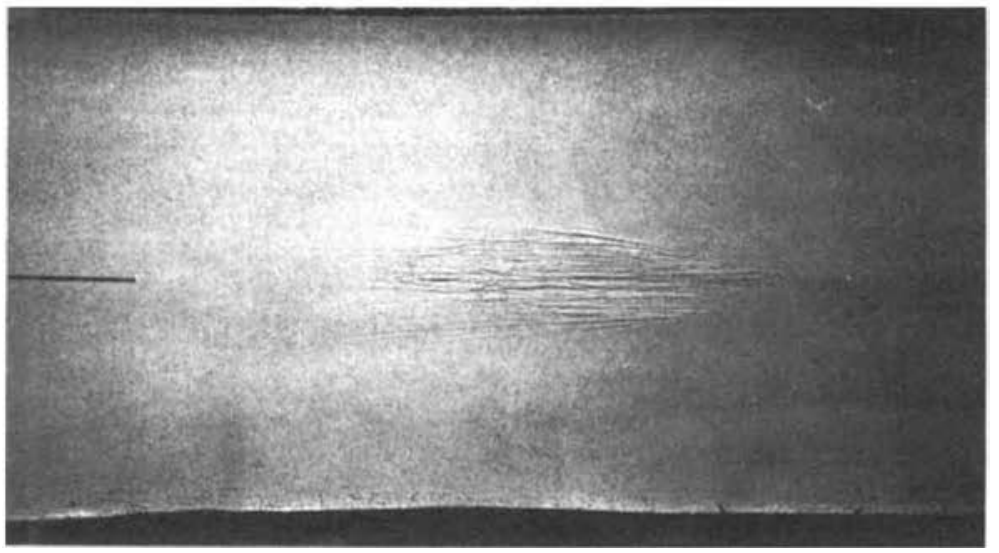

FIGURE 2. Shadowgraph visualization (side view) of the evolution of the turbulent region. The photographs were taken at $(a) t=1 \mathrm{~s},(b) 2 \mathrm{~s},(c) 3 \mathrm{~s},(d) 4 \mathrm{~s},(e) 7 \mathrm{~s},(f) 20 \mathrm{~s}$. The experimental parameters: $Q=2.5 \mathrm{ml} \mathrm{s}^{-1}, \delta t=0.2 \mathrm{~s}$ and $N=2.7 \mathrm{rad} \mathrm{s}^{-1}$, the tank dimensions $(h \times l \times w)$ are $40 \times 30 \times 15 \mathrm{~cm}$. The length of the nozzle $(5 \mathrm{~cm})$ provides the lengthscale.

trajectory. The present study, however, focuses on symmetric dipoles with a net circulation approximately equal to zero.

Qualitative information about the collapse process and the transition from three- to two-dimensional turbulence was obtained by shadowgraph visualization. Figure 2 
(a)

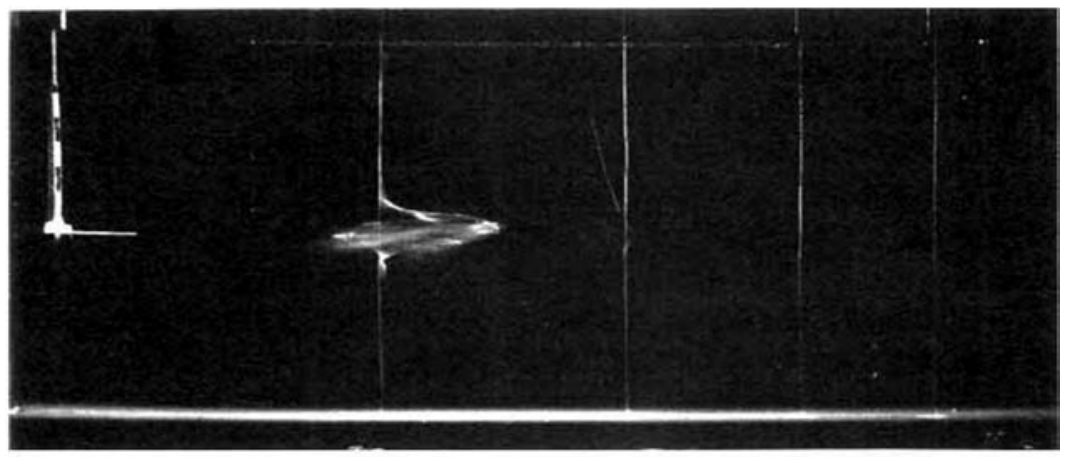

(b)

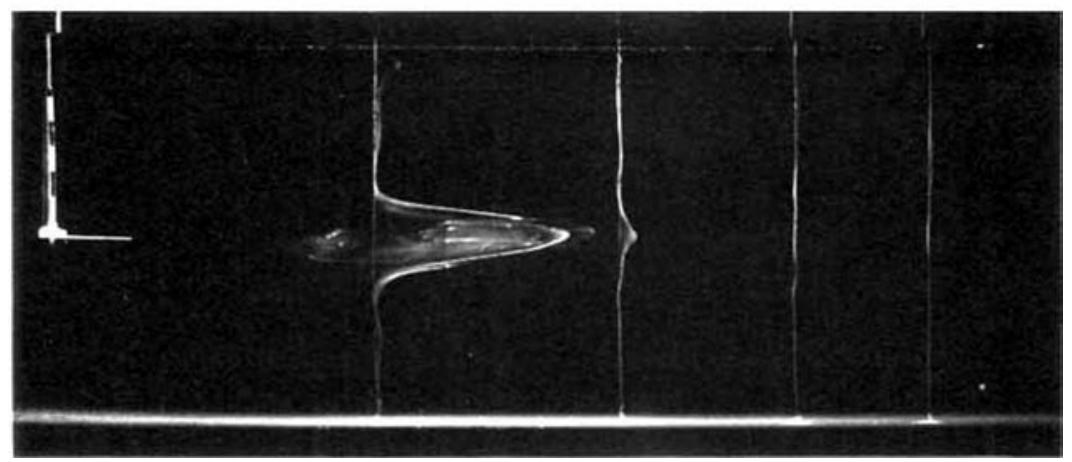

FIGURE 3. Photographs showing the horizontal displacement of a vertical dye line positioned on the dipole's symmetry-axis due to the translating vortex structure. The photographs were taken at (a) $t=40 \mathrm{~s}$ and (b) $80 \mathrm{~s}$. The experimental parameters: $Q=2 \mathrm{ml} \mathrm{s}^{-1}, \delta t=0.5 \mathrm{~s}$ and $N=2.05 \mathrm{rad} \mathrm{s}^{-1}$. The bars on the strip each measure $2 \mathrm{~cm}$.

contains a sequence of side-view shadowgraph photographs, which clearly reveal the flow evolution. In figure $2(a)$ we observe that, just after the injection, the turbulence is mainly concentrated at the front of the region, where the mixed fluid of the jet appears to be blocked by the ambient fluid, as is apparent from the blunt nose of the intrusion. The next photograph (figure $2 b$ ) shows the situation just after the onset of the collapse: while the inner parts of the flow region still contain large overturning eddies, at its edge the vertical motions are significantly suppressed by buoyancy forces. Gradually, the overturning motions in the interior of the patch vanish (figure $2 c, d$ ). Af ter the collapse the turbulence is 'fossilized' (see Gibson 1980), and the subsequent shadowgraph pictures show typical horizontal striations (figure $2 e, f$ ).

Internal waves, generated during the collapse, cause perturbations in the ambient basic linear density stratification, which show up as rays of darker and lighter grey tones in the shadowgraph pictures, see figure $2(b-e)$. These spatial variations in the grey tones can be interpreted as areas where the second (spatial) derivative of the density is different from its surroundings. The wave rays visible on the photographs are thus the lines of constant phase associated with the internal wave motion. Their orientation provides information about the direction in which energy is transported, whereas the grey tones provide a measure for the amplitude of the wave motion. Larger wave amplitudes induce larger gradients in grey tones.

The structure of the ray pattern clearly illustrates that kinetic energy is also radiated 
away in the vertical direction, from an apparent point in the centre of the collapsing region. The waves continue to exist until the collapse is completed (figure $2 d, e, f$ ). From the subsequent ray patterns it is observed that the ray angles with the horizontal gradually decrease. A similar change of wave direction has been observed by $\mathrm{Wu}(1969)$ in experiments on a (steady) intrusion in a stratified fluid. From the sequence of shadowgraph pictures it is seen that throughout the flow evolution the striations are concentrated within a relatively compact region, which more or less coincides with the dyed region as seen in the experiment of figure 1 . Also, it is observed that the striations become longer over time, finally being confined within an oval-shaped region, see figure $2(f)$.

Although the motion in the eventual dipolar vortex is planar, it is not exactly twodimensional: the horizontal motion associated with the dipole translating through the quiescent ambient fluid being confined within a thin pancake-shaped region implies vertical shear at its upper and lower sides. In order to examine the vertical distribution of the horizontal motion due to the translating dipole, experiments were carried out in which vertical dye lines were created at several positions, both upstream of and inside the dipole. The dye lines were produced by applying the so-called precipitation method (Honji, Taneda \& Tatsuno 1980). Typical results obtained by this visualization technique are presented in figure 3. The distortions of the dye lines visible in the photographs clearly show that the horizontal motion due to the moving dipole is confined to a relatively thin layer, and that considerable shear occurs at its upper and lower boundaries. For the (characteristic) case shown in figure 3 , the layer thickness $2 \sigma$ is estimated to be $5 \mathrm{~cm}$. With a maximum horizontal velocity of $0.5 \mathrm{~cm} \mathrm{~s}^{-1}$, this implies a magnitude of the vertical shear of the order $\Delta U / \sigma=0.2 \mathrm{~s}^{-1}$. The buoyancy frequency is $N=1.5 \mathrm{rad} \mathrm{s}^{-1}$ in this case, so that the bulk Richardson number $R i=N^{2} /(\Delta U / \sigma)^{2} \geqslant$ 50 for the sheared flow. This suggests that the motion in the shear layer is stable in the sense that no overturning motions are to be expected. This is confirmed by the observations. During the evolution (and decay) of the dipolar vortex, the magnitude of the shear decreases because of both the increase in layer thickness and the decrease of the dipole's translation speed. The Richardson number thus gradually increases, implying that the shear-layer flow becomes more and more stable.

In a similar experiment dye was generated by the precipitation method at a number of points (at regular distances of approximately $1 \mathrm{~cm}$ ) on a solder wire that was placed vertically in the flow. Horizontal streaklines were formed by the advection of the dye within one of the vortices of the dipole, and vertical motions could be detected from the vertical displacement of the streak over a certain horizontal distance. A typical example of these dye streaks is shown in figure 4. Although some diffusion of the dye took place, within the vortex centres vertical displacements also appear to be negligible.

In order to visualize (and actually measure) the horizontal flow in the horizontal plane of the dipole, both inside it and outside, streak photographs were taken of tracer particles floating in that particular plane. A typical example of such a streak photograph is presented in figure 5. In this experiment the dipolar structure is also visible in the dye distribution, which allows a comparison to be made between both types of visualization. For this purpose the injected fluid was dyed with esculine, which became fluorescent when illuminated by UV light. During the exposure period, required for the streaks to be visible, there was a UV-flash during a relatively short time interval. The dye can be thought of as representing the vortical flow region in a frame co-moving with the dipole, whereas the streaks visualize the entire flow field induced by the translating dipole relative to the fixed laboratory frame. At the edge of the dye pattern the particle streaks are observed to intersect under a certain angle, in particular 


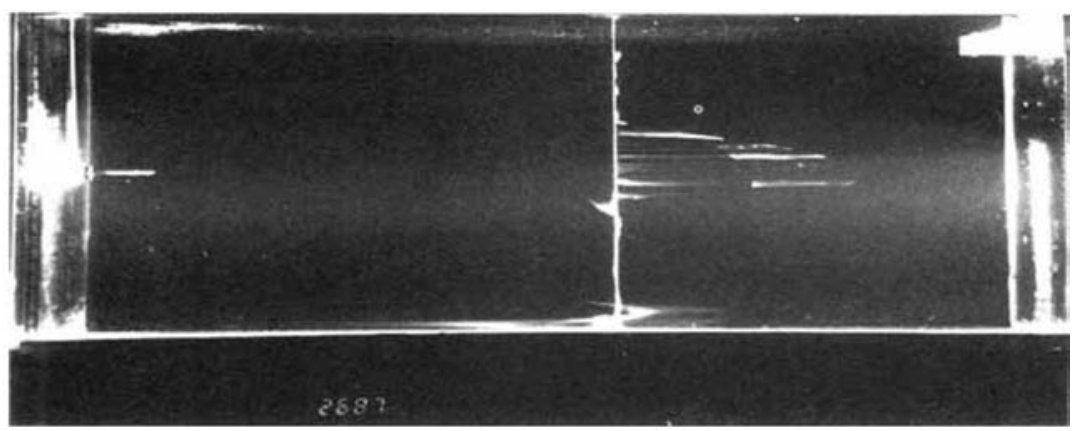

FIgURE 4. Photograph showing the advection of dye by one of the vortices of the dipolar structure. The dye is created in points (at distances of about $1 \mathrm{~cm}$ ) on a solder wire. The picture was taken at $t=145 \mathrm{~s}$. Experimental parameters: $Q=2 \mathrm{ml} \mathrm{s}^{-1}, \delta t=1 \mathrm{~s}$ and $N=1.7 \mathrm{rad} \mathrm{s}{ }^{1}$. The length of the nozzle $(5 \mathrm{~cm})$ provides the lengthscale.

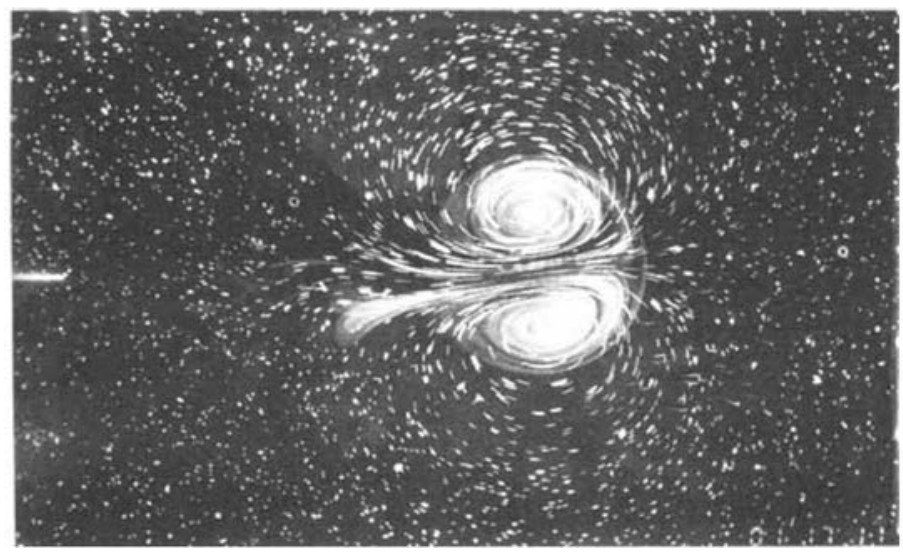

FIGURE 5. Combination of streak and dye visualization of the dipolar vortex. The picture was taken at $t=180 \mathrm{~s}$. During the exposure period of $22 \mathrm{~s}$, the UV-sensitive dye (esculine) was illuminated with a UV lamp for a period of $0.5 \mathrm{~s}$. Experimental parameters: $Q=6 \mathrm{ml} \mathrm{s}^{-1}, \delta t=0.5 \mathrm{~s}$ and $N=$ 2. $1 \mathrm{rad} \mathrm{s}^{-1}$.

at the front. This effect is due to the different frames of reference of the dye and the streak visualizations, as just mentioned. The exterior flow pattern visible in figure 5 is not dissimilar to that due to a circular cylinder moving steadily along a straight line through a fluid, seen in a fixed frame. In view of this, the flow can be considered as consisting of a closed region (more or less delineated by the dye distribution) containing vorticity, surrounded by a region of potential flow. For descriptional purposes it is convenient to consider the (interior and exterior) flow in a frame comoving with the dipole. Once the streak photograph is digitized and the corresponding velocity field is determined (see $\S 5$ ), it is easy to carry out this transformation by simply subtracting the dipole translation speed.

In order to investigate the vertical distribution of the horizontal velocity, additional experiments were performed in which the dipolar vortex (visualized by dye) was created below the particle level by injecting at a lower level than that of the particles. Initially, the dipole is confined in a thin layer so that the particles are not affected by the dipolar flow, but owing to vertical diffusion the dipolar structure grows in thickness so that the vortical motion eventually reaches the level of the particles. This is clearly observed in the sequence of photographs presented in figure 6 : in the initial stage 
(a)

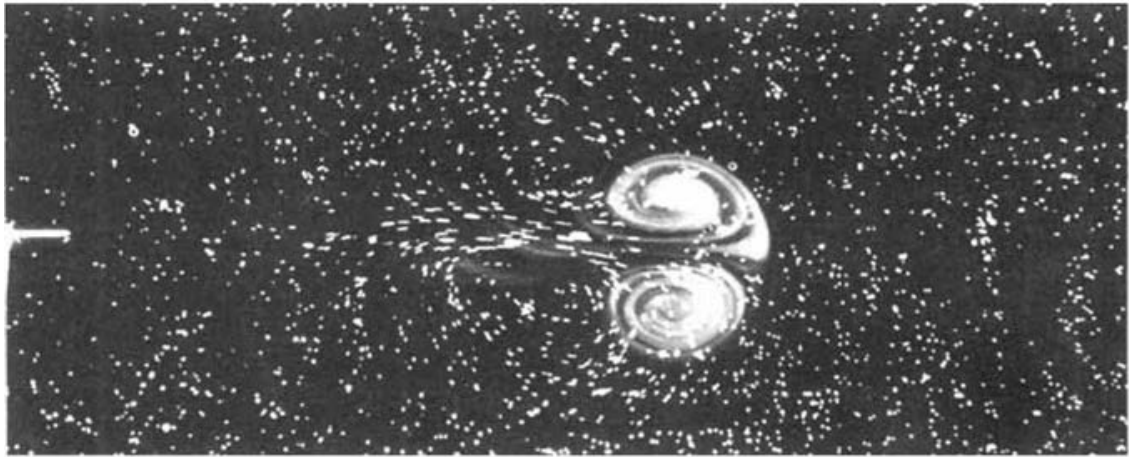

(b)

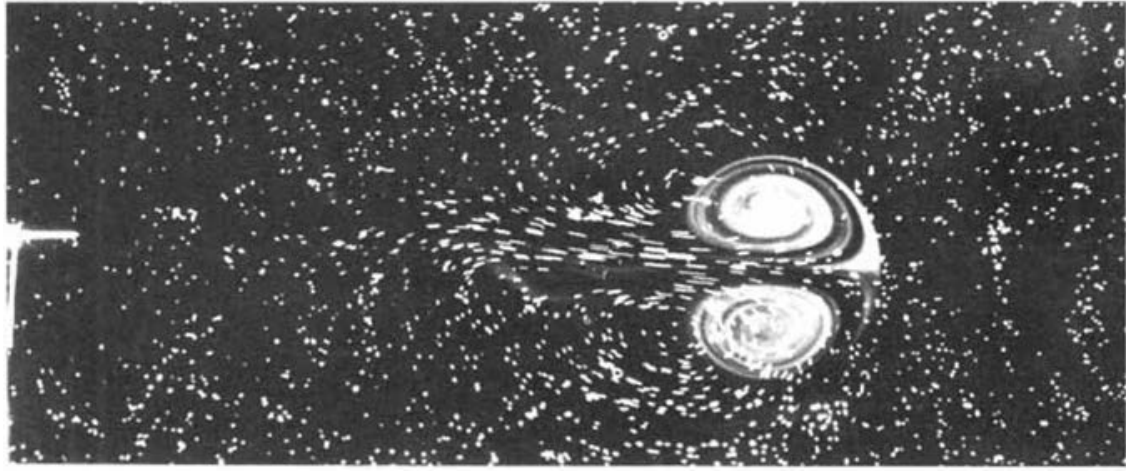

(c)

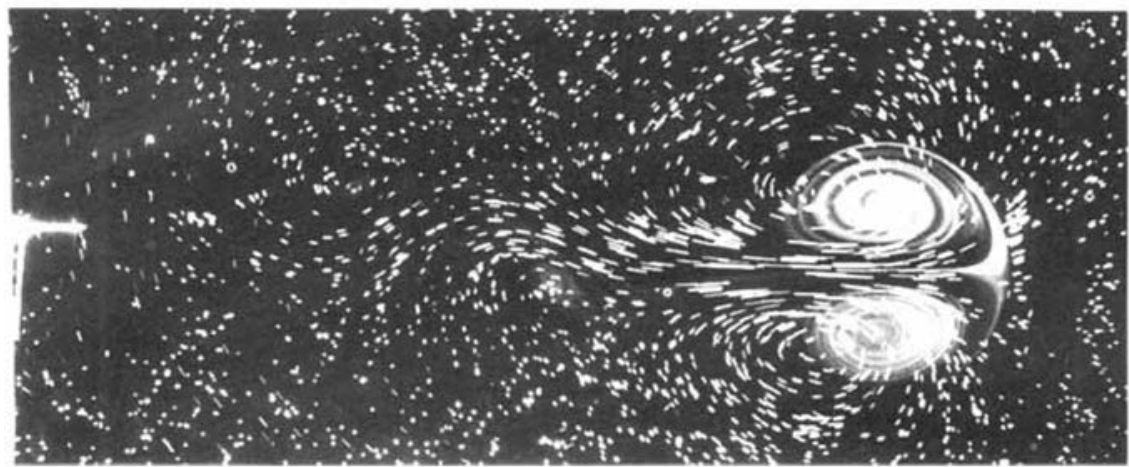

FiguRE 6. Visualization as in figure 5, but here the dye-visualized dipole is created $3.5 \mathrm{~cm}$ below the level of the particles. The photographs were taken at $(a) t=95 \mathrm{~s},(b) 140 \mathrm{~s},(c) 190 \mathrm{~s}$. Experimental parameters: $Q=10 \mathrm{ml} \mathrm{s}^{-1}, \delta t=0.3 \mathrm{~s}$ and $N=2.1 \mathrm{rad} \mathrm{s}^{-1}$. The length of the nozzle $(5 \mathrm{~cm})$ provides the lengthscale.

(figure $6 a$ ) the motions of the particles indicate a more or less unif orm horizontal flow at this higher level behind the 'midplane' dipolar vortex structure visible by dye. As time proceeds, the dipole thickness and the vortex motion become visible in the streak pattern (figure $6 b$ ). Note that the centres of the vortical streak pattern are visible some distance behind the dyed mid-plane dipolar structure. This distance decreases in time, as a result of the further thickening of the vortical flow region due to diffusion, see figure $6(c)$.

An important question concerns the topology of the vortex lines of these vortex structures, which behave as isolated vorticity regions in an otherwise quiescent (and 


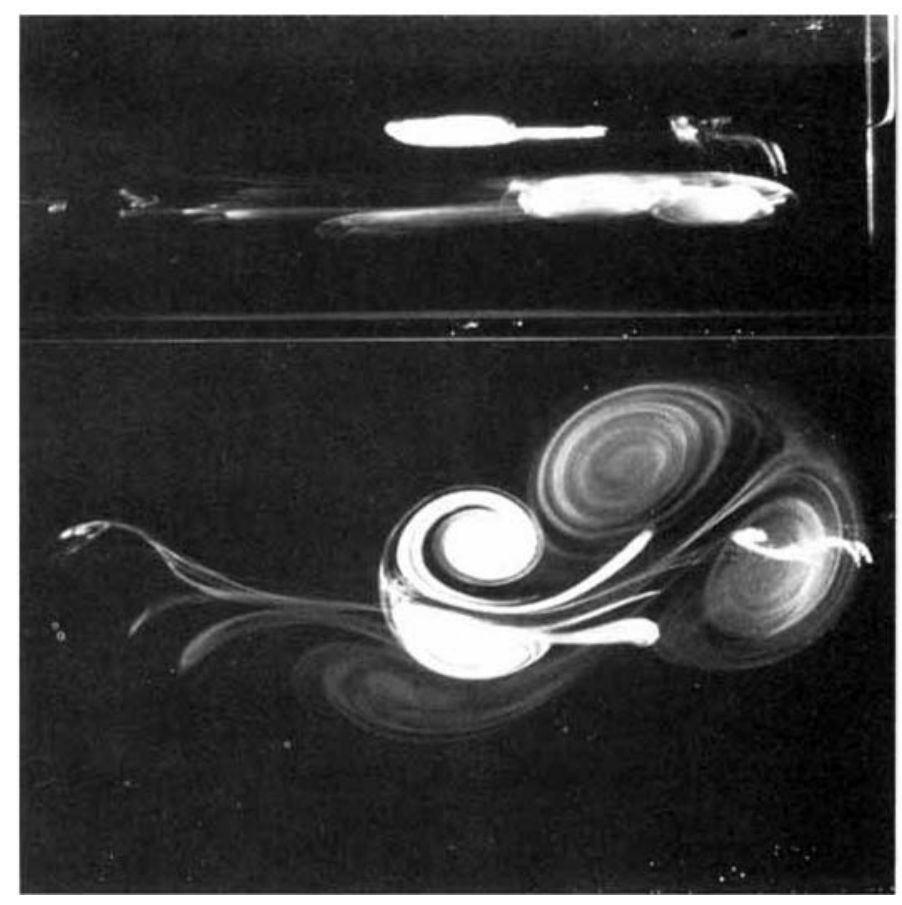

Figure 7. Combined top-view and side-view photograph (as in figurc 1) of two dipoles moving horizontally through the fluid at different levels. The experimental parameters: $Q=6 \mathrm{ml} \mathrm{s}{ }^{-1}, \delta t=$ 0.1 s (large dipole) and $Q=1 \mathrm{ml} \mathrm{s}{ }^{-1}, \delta t=0.5 \mathrm{~s}$ (small dipole), with $N=1.7 \mathrm{rad} \mathrm{s}^{-1}$.

thus irrotational) ambient fluid. Because of the symmetry about the horizontal midplane, the vortex lines will be directed vertically at the intersection with this horizontal plane. The vertical distribution of the horizontal motion, as illustrated by figure 3 , and the backward-shif ted vortex centres at lower and higher levels (figure $6 h, c$ ) imply that the vortex lines are bent some distance from the mid-plane and presumably close some distance behind the dipole at a level where the horizontal shear is dominant and vorticity is mainly directed horizontally.

The fact that the motion associated with the moving dipolar vortex is concentrated in a relatively thin layer of fluid is nicely illustrated by the photograph shown in figure 7. In this experiment two dipoles were produced at different levels, which were observed to move over each other without affecting the other vortex: although both vortex dipoles were somewhat asymmetric, their motions were completely disconnected.

In vicw of the observed vertical distribution of the horizontal flow, the dipolar flow structure can to a good approximation be considered as a slice of finite thickness in which the flow is two-dimensional, with the vorticity lines directed vertically. It will be shown in the next section that it is possible to scek a theoretical description of the vortex dipole in terms of two-dimensional flow theory.

\section{Theoretical considerations on the vortex dipole}

First, a scaling analysis is presented by which it is found that to leading order the dipolar flow structure can be considered as quasi-two-dimensional. This analysis is followed by a concise description of the Lamb-Chaplygin dipole model and some of its general properties. 


\subsection{Scaling analysis}

In order to determine the terms that govern the flow, a scaling analysis based on that of Riley, Metcalfe \& Weissman (1981) and Lilly (1983) is presented first. Riley et al. (1981) found that for an initially isotropic turbulent flow buoyancy-dominated flow fields - to lowest order - can be decomposed into vertical motions, induced by internal gravity waves, and stratified (quasi-two-dimensional) turbulence. We will closely follow their scaling analysis.

The flow is assumed to satisfy the Boussinesq approximation for the Navier-Stokes equations, and the continuity equation, i.e.

$$
\begin{gathered}
\frac{\partial \boldsymbol{u}}{\partial t}+(\boldsymbol{u} \cdot \boldsymbol{\nabla}) \boldsymbol{u}=-\frac{1}{\bar{\rho}} \nabla p^{\prime}+\boldsymbol{g} \frac{\rho^{\prime}}{\bar{\rho}}+\nu \nabla^{2} \boldsymbol{u}, \\
\boldsymbol{\nabla} \cdot \boldsymbol{u}=0,
\end{gathered}
$$

leading to

$$
\frac{\partial \rho^{\prime}}{\partial t}+(\boldsymbol{u} \cdot \nabla) \rho^{\prime}+w \frac{\mathrm{d} \rho_{0}}{\mathrm{~d} z}=0,
$$

where $\boldsymbol{u}=(u, v, w)$ is the velocity vector with components in the directions of the Cartesian coordinates $x, y, z$, with $z$ in the vertical, $\bar{\rho}=\rho\left(z_{0}\right)$ is the constant reference density, $\rho^{\prime}$ the density deviation from the ambient density $\rho_{0}=\rho(z), p^{\prime}$ the pressure deviation from the ambient pressure and $g=(0,0, g)$ the gravity vector. Mass diffusion of salt is very slow and its effect is assumed negligible. The horizontal velocity is scaled by the typical horizontal translation velocity scale $U_{0}$, while the horizontal and vertical lengthscales are $D$ and $2 \sigma$, respectively, and time is scaled with the advective timescale $D / U_{0}$. Horizontal pressure derivatives are assumed to be of the same order as the accelerations, implying that the scale for the pressure is $\bar{\rho} U_{0}^{2}$. Since the vertical motions are very small, the $z$-component of (1) describes to a good approximation a hydrostatic balance, implying that the vertical pressure-gradient term $(1 / \overline{\boldsymbol{\rho}})\left(\partial p^{\prime} / \hat{c} z\right)$ and the buoyancy term $g \rho^{\prime} / \bar{\rho}$ are of the same order. The scale of the density variation $\rho^{\prime}$ is thus $\overline{\boldsymbol{\rho}} U_{0}^{2} /(2 \sigma g)$. The magnitude of the vertical velocity, $w$, is found by assuming that in (3) the term $c \rho^{\prime} / \partial t$ and the stratification term $w \mathrm{~d} \rho_{0} / \mathrm{d} z$ are of the same order. This yields the scale $U_{0} \alpha /(4 R i)$ for the vertical velocity, where $R i$ is the Richardson number as defined in $\S 3$ and $\alpha=2 \sigma / D$ is the ratio of the lengthscales. With the scales

$$
\begin{gathered}
u \sim U_{0} u^{*}, \quad w \sim \frac{U_{0} \alpha}{4 R i} w^{*}, \quad t \sim \frac{D}{U_{0}} t^{*}, \quad \rho^{\prime} \sim \frac{\bar{\rho} U_{0}^{2}}{2 \sigma g} \rho^{\prime *}, \\
(x, y) \sim\left(D x^{*}, D y^{*}\right) \quad \text { and } z \sim 2 \sigma z^{*}
\end{gathered}
$$

one obtains after omitting the asterisks

$$
\begin{gathered}
\frac{\partial \boldsymbol{u}_{h}}{\partial t}+\left(\boldsymbol{u}_{h} \cdot \nabla_{h}\right) \boldsymbol{u}_{h}+\frac{w}{4 R i} \frac{\partial \boldsymbol{u}_{h}}{\partial z}=-\nabla_{h} p^{\prime}+\frac{1}{R e_{h}} \nabla_{h}^{2} \boldsymbol{u}_{h}+\frac{1}{\alpha R e_{v}} \frac{\partial^{2} \boldsymbol{u}_{h}}{\partial z^{2}}, \\
\boldsymbol{\nabla}_{h} \cdot \boldsymbol{u}_{h}+\frac{1}{4 R i} \frac{\partial w}{\partial z}=0, \\
\frac{\partial \rho^{\prime}}{\partial t}+\left(\boldsymbol{u}_{h} \cdot \nabla_{h}\right) \rho^{\prime}+\frac{w}{4 R i} \frac{\partial \rho^{\prime}}{\partial z}+w=0,
\end{gathered}
$$

where $\boldsymbol{u}_{h}$ the horizontal velocity scale, $w$ the vertical velocity and $\boldsymbol{\nabla}_{h}$ the gradient operator acting in the horizontal directions. The factor 4 arises because of the 
definition of $R i$, which is based on the shear $U / \sigma$. The influence of the shear term in (4) can be represented by the ratio $4 R i /\left(\alpha R e_{v}\right)=\nu N^{2} D / U^{3}$. In a very few experiments, for a minimal value of $N$ and a maximum value of $U$, this ratio had a value of $O(1)$ just after the dipole formation. For the majority of the experiments, however, this ratio had an initial value significantly larger than $O(1)$. Besides, owing to the decay of the dipole's translation velocity $U$, this ratio rapidly increases and soon becomes one or two orders of magnitudes larger than the initial value. This implies that the shear term in (4) can to leading order be neglected. The horizontal momentum and continuity equations are then

$$
\begin{gathered}
\frac{\partial \boldsymbol{u}_{h}}{\partial t}+\left(\boldsymbol{u}_{h} \cdot \nabla_{h}\right) \boldsymbol{u}_{h}=-\nabla_{h} p^{\prime}+\frac{1}{R e_{h}} \nabla_{h}^{2} u_{h}+\frac{1}{\alpha R e_{v}} \frac{\partial^{2} \boldsymbol{u}_{h}}{\partial z^{2}}, \\
\boldsymbol{\nabla}_{h} \cdot \boldsymbol{u}_{h}=0,
\end{gathered}
$$

while in the vertical direction the hydrostatic balance is assumed. These equations describe the horizontal vortex motion that is decoupled from the vertical motions. Vertical velocities are negligible, so that the (three-dimensional) vorticity $\omega$ for this flow can be written as

$$
\boldsymbol{\omega}=\boldsymbol{\nabla} \times \boldsymbol{u}=\left(-\frac{\partial v}{\partial z}\right) \boldsymbol{i}+\left(\frac{\partial u}{\partial z}\right) j+\left(\boldsymbol{\nabla}_{h} \times \boldsymbol{u}_{h}\right) \boldsymbol{k},
$$

with $(\boldsymbol{i}, \boldsymbol{j}, \boldsymbol{k})$ the unit vectors in the $(x, y, z)$ directions. Thus, $\boldsymbol{\omega}$ will be parallel to $\boldsymbol{k}$ only for $\mathrm{\partial} / \partial z=0$, i.e. at the level $z=0, \partial \boldsymbol{u}_{h} / \partial z=0$, while for $|z|>0$ horizontal vorticity is associated with the shear. With the stream function $\psi$ defined by $\boldsymbol{u}_{h}=-\boldsymbol{k} \times \boldsymbol{\nabla} \psi$ one obtains the $z$-component of the vorticity

$$
\frac{\partial \omega_{z}}{\partial t}+J\left(\omega_{z}, \psi\right)=\frac{1}{R_{h}} \nabla_{h}^{2} \omega_{z}+\frac{1}{\alpha R e_{v}} \frac{\partial^{2} \omega_{z}}{\partial z^{2}},
$$

where

$$
\omega_{z}=-\nabla_{h}^{2} \psi
$$

and $J$ is the Jacobian defined by

$$
J(\omega, \psi)=\frac{\partial \omega}{\partial x} \frac{\partial \psi}{\partial y}-\frac{\partial \omega}{\partial y} \frac{\partial \psi}{\partial x} .
$$

As shown in the observations the horizontal Reynolds number $R e_{h}$ is much larger than the vertical Reynolds number $R e_{v}$, indicating that the evolution of the horizontal flow is mainly due to vertical diffusion. For large $R e$-values (9) resembles the inviscid vorticity equation of purely two-dimensional flow for which quasi-steady solutions are governed by $J\left(\omega_{z}, \psi\right)=0$. When choosing such a quasi-steady solution as a basic state, (9) reduces to leading order to

$$
\frac{\partial \omega_{z}}{\partial t}=\frac{1}{\alpha \operatorname{Re}_{v}} \frac{\partial^{2} \omega_{z}}{\partial z^{2}}
$$

which describes a purely diffusive decay. Because horizontal vortex motions are to lowest order decoupled from vertical motions and internal waves - with, consequently, radiation of energy - are negligible, vertical vorticity $\omega_{2}$ decays mainly due to this vertical diffusion.

For the vortex dipoles considered here, the viscous timescale is large in comparison with the advective timescale $L / U$ based on the translation speed $U$ and the dipole size 
$L$, so that the viscous term in (9) can to a good approximation be neglected, which yields the vorticity equation for a purely two-dimensional flow:

$$
\frac{\partial \omega}{\partial t}+J(\omega, \psi)=0
$$

in which the subscript $z$ has been dropped. In a frame moving with the dipole, the vortex structure is stationary, and is governed by $J\left(\omega^{\prime}, \psi^{\prime}\right)=0$. The prime denotes the correction for the translation of the dipole, i.e. $\omega^{\prime}=\omega$ and $\psi^{\prime}=\psi-U_{x} y+U_{y} x$ for a dipole translating (along a straight line) at speed $\left(U_{x}, U_{y}\right)$. In what follows we will refer only to the corrected vorticity $\omega^{\prime}$ and stream function $\psi^{\prime}$, and for convenience the primes will be omitted from now on. The vanishing Jacobian implies that any functional relationship $F$ between $\omega$ and $\psi$,

is satisfied.

$$
\omega=F(\psi)
$$

\subsection{The vortex dipole model}

A useful model for a two-dimensional dipolar vortex with continuously distributed vorticity on a circular area was described by Lamb (1932) and also by Batchelor (1967). In a recent paper by Meleshko \& van Heijst (1994) it is pointed out that a similar, more general, dipole solution was formulated independently by the Russian scientist $\mathrm{S}$. A Chaplygin in a paper that was published as early as 1903. In the literature the analytical dipole solution is usually referred to as the 'Lamb dipole'. In view of the recently obtained evidence about Chaplygin's contributions to the subject, however, the name 'Lamb-Chaplygin dipole' seems more appropriate, and in the present paper we will therefore adopt this name. In order to obtain an analytical solution of (10), Lamb and Chaplygin assumed a linear relationship

$$
\omega=k^{2} \psi,
$$

with $k$ some constant, to be valid on a circular region with radius $r=a$. Outside the circle, the flow was taken as irrotational. The Lamb-Chaplygin dipolar solution is given by

$$
\left.\begin{array}{ll}
\psi=-\frac{2 U_{0}}{k J_{0}(k a)} J_{1}(k r) \sin \theta & \text { for } \quad r \leqslant a \\
\psi=U_{0}\left(r-\frac{a^{2}}{r}\right) \sin \theta \quad \text { for } \quad r \geqslant a,
\end{array}\right\}
$$

with $U_{0}$ the uniform fluid velocity at infinity (in the fixed frame this is the dipole's translation speed), $\theta$ the azimuthal coordinate measured from the dipole's axis $(\theta=0$, $\pi$ is the symmetry axis) and $J_{i}$ the $i$ th-order Bessel function of the first kind. Continuity of the interior and exterior solutions on $r=a$ requires

$$
k a=3.831,
$$

being the first zero of $J_{1}$. Obviously, the radius of the Lamb-Chaplygin dipole depends on the wavenumber $k$ that determines the proportionality constant between $\omega$ and $\psi$, see (14).

It is useful, for later comparison purposes, to mention a few general characteristics of this dipole model, which can be derived from (15) straightforwardly. First, the maximum velocity (within the framework of the stationary dipole) is found on the symmetry axis at $r=0$ and is given by $U_{m \boldsymbol{a} x}=2.49 U_{0}$. Apparently, in the fixed laboratory frame this speed is $U_{\text {max }}^{*}=U_{m \frac{15}{15}}+U_{0}=3.49 U_{0}$. Furthermore, the extremal 
vorticity in the dipole is equal to $\omega_{\max }=\left|\omega_{\min }\right|=2.89 \mathrm{k} U_{0}$; the distance between the points of minimum and maximum vorticity is $d=0.96 a$. Another useful 'distance' in particular when characterizing dipolar vortices from streak photographs like the one shown in figure 5-is the separation distance between the two apparent 'centres' of rotational motion, which for the Lamb-Chaplygin dipole is found to be $d_{v c}=1.234 a$. This distance $d_{v c}$ (henceforth referred to as the distance between the virtual vortex centres) and the maximum velocity $U_{\max }^{*}$ can be measured directly from streakline photographs, and are therefore convenient quantities.

Other useful integral quantities are the circulation $\Gamma$ (per dipole half : $\Gamma_{+}$and $\Gamma_{-}$), the kinetic energy $E$ and the fluid impulse $P$ in the direction of translation within the dipole region (both per unit mass and per unit length perpendicular to the plane of flow):

$$
\begin{gathered}
\Gamma=\Gamma_{+}=\left|I_{-}\right|=\int_{0}^{\pi} \int_{0}^{a} \omega r \mathrm{~d} r \mathrm{~d} \theta=6.83 U_{0} a, \\
E=\int_{0}^{2 \pi} \int_{0}^{a}\left(u^{2}+v^{2}\right) r \mathrm{~d} r \mathrm{~d} \theta=4 \pi U^{2} a^{2}, \\
P=2 \int_{0}^{\pi} \int_{0}^{a} \omega r^{2} \sin \theta \mathrm{d} r \mathrm{~d} \theta=2 \pi U_{\bullet} a^{2} .
\end{gathered}
$$

Alternatively, these results can also be expressed in terms of the wavenumber $k$ rather than the radius $a$, by applying the dispersion relation (16). By combination of (17) and (19) one can obtain the following expression for the distance $d$ between the centroids of the vorticity of the Lamb-Chaplygin dipole:

$$
d=\frac{P}{\Gamma}=2 \pi \frac{a}{6.83} .
$$

By comparison, a point-vortex dipole with vortices of strengths $\pm \Gamma$ placed a distance $d$ apart given by (20) will have a translation speed

$$
U=\frac{\Gamma}{4 \pi^{2} a / 6.83}=\frac{\Gamma}{5.78 a},
$$

which is slightly larger than the translation speed of the Lamb-Chaplygin dipole, see (17). Although some attempts have been made to construct vortex dipole models with distributed vorticity (see e.g. Pierrehumbert 1980 who considered symmetric arrangements of two compact uniform-vorticity patches of non-circular shapes), at present no other theoretical models of a continuous dipolar vorticity distribution on a compact (circular) area are available. In the following section the observed and measured characteristics of the dipolar vortices in a stratified fluid will be compared with the Lamb-Chaplygin model.

\section{Measurements of the dipole characteristics}

In this section quantitative measurements of the flow field due to the dipolar vortex will be presented. The majority of the experiments concerned dipolar vortices emerging after the collapse of a turbulent region created by turbulent injection, as described in $\S 3$, and the main results will be presented first. In addition, dipolar vortices were also generated by laminar injection, and their measured characteristics will also be discussed for comparison. Finally, attention will be given to the behaviour of the dipole while decaying, i.e. on a large timescale. 


\subsection{Turbulent-injection dipoles}

\subsubsection{Measurement of the flow field quantities}

Quantitative information about the evolution of the dipolar flow field was obtained by digitization of the streak photographs. The digitization implies locating the starting point and end point of each streak (or of a representative sample of the streaks). This procedure was carried out manually on a digitization tablet, which was connected to a personal computer. After calculating the patch lengths from the coordinates of the end points of the streaks, and by dividing by the photographic exposure time, one obtains for each streak photograph a velocity vector field such as shown in figure $8(a)$. In most cases approximately 150 to 250 streaks were selected in such a way that one obtains a digitized velocity field with an even distribution of vectors.

Next, the digitized velocity field was fitted by cubic splines, which allows further numerical interpolation onto, for example, a rectangular $n \times m$ grid. The cubic splines used here have the useful properties of avoiding spurious oscillations, being independent of the chosen coordinate axes $x$ and $y$, and keeping the exact values at the measured points (for a more extensive description, see Nguyen Duc \& Sommeria, 1988). In all cases described in the present paper, a rectangular grid with dimensions $30 \times 30$ was used, positioned in such a way that it completely covers the flow area of interest. The interpolated velocity field $\boldsymbol{v}(\boldsymbol{x})$ of the digitized velocity field of figure $8(a)$ is presented in figure $8(b)$, which shows a smooth dipolar pattern. By numerical differentiation of the interpolated velocity field according to

$$
\omega=\frac{\partial v}{\partial x}-\frac{\partial u}{\partial y}
$$

one obtains the value of the vorticity $\omega$ in each grid point. Figure 8(c) shows a contour plot of the vorticity distribution corresponding with the dipolar velocity field of figure 8 (b). It can be clearly observed that the vorticity is concentrated in two compact areas, in which it has opposite signs. Moreover, the contour plot reveals a continuous distribution of $\omega$ within each patch. Next, the stream function $\psi$ is calculated in each grid point by numerically solving the Poisson-type equation (10). For the particular experiment discussed here, the resulting $\psi$-distribution is shown by the contour plot of figure $8(d)$.

As discussed in $\S 4$, it is more convenient to describe the flow field in a frame of reference moving with the dipole. For a dipole translating with velocity $\left(U_{x}, U_{y}\right)$ this requires the following transformation: $\psi^{\prime}=\psi-U_{x} y+U_{y} x$, while the vorticity remains unchanged, i.e. $\omega^{\prime}=\omega$. A typical result of such a translation correction is shown in the $\psi$-contour plot of figure $8(e)$. This graph clearly reveals the existence of a more or less circular streamline inside which a dipolar flow pattern is visible; the flow exterior to the closed streamline very much resembles that of an irrotational flow around a cylinder.

In the initial stages of the work reported here, the Poisson equation (10) was numerically solved on the square $30 \times 30$ grid by taking $\psi=0$ as boundary condition on the grid edge. However, in a number of cases the computational domain was found to be too small compared to the dipole size, and imposing the boundary condition $\psi=0$ led to incorrect results: one thus obtains the $\psi$-field associated with a dipole moving in a narrow channel with finite length. In order to eliminate this artifact, the stream-function values on the boundary are calculated separately from the velocity vectors at the edge grid points, and the points of zero velocity (reference points for the interpolation spline functions) were placed a distance apart of approximately three 

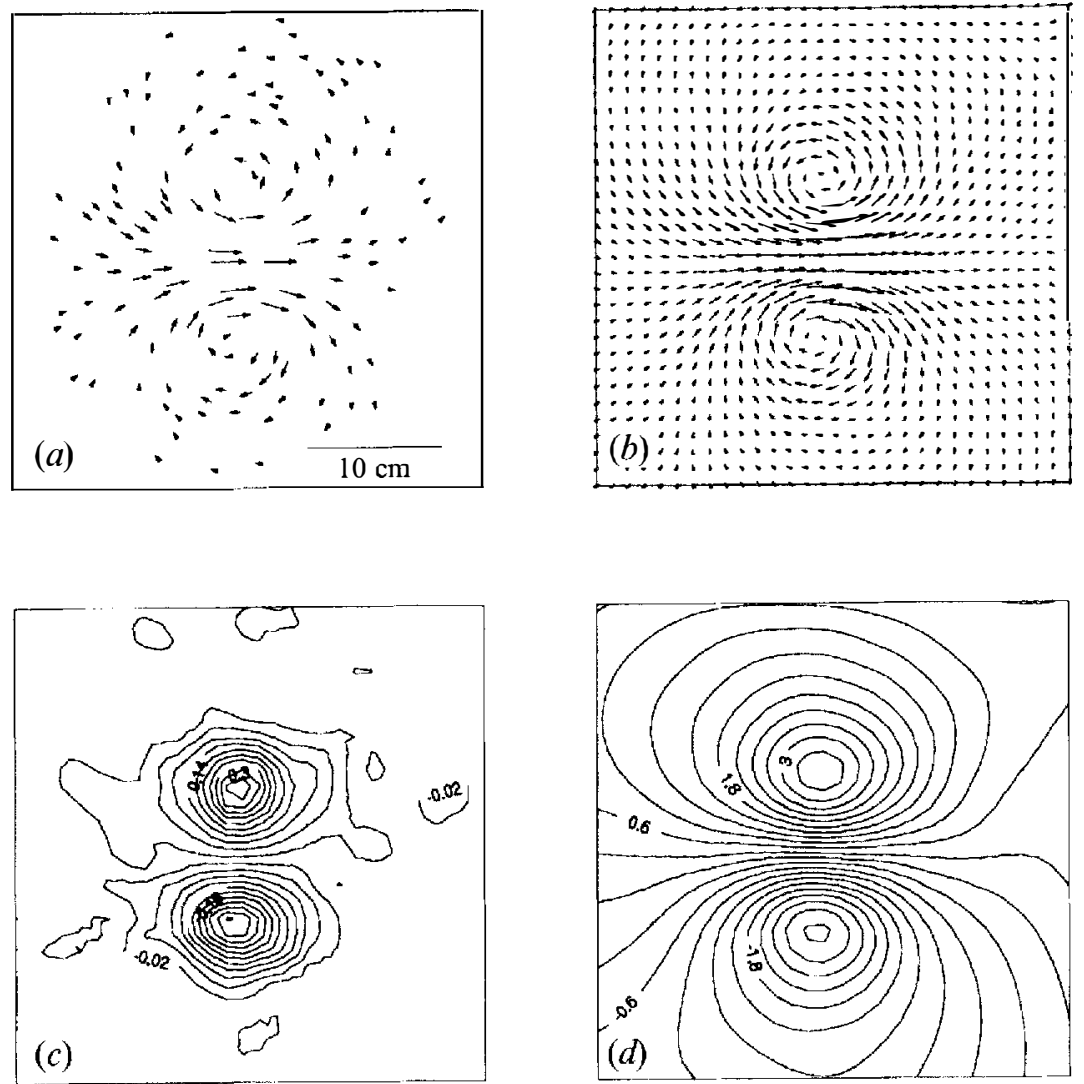

$\omega$

$\psi$

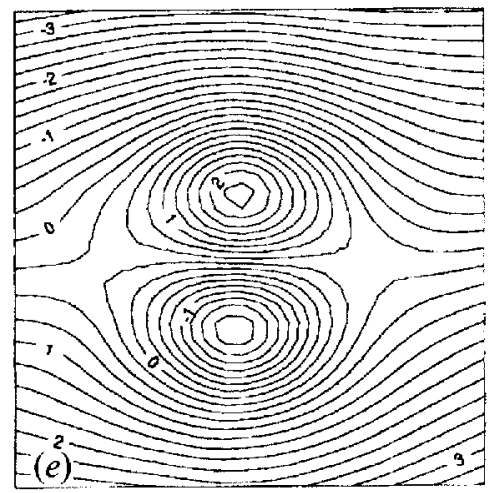

$\psi-c(x, y)$

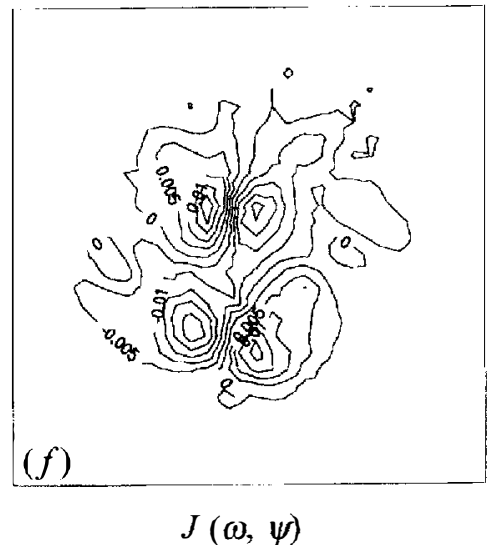

Fugure 8. A typical digitized flow field $(a)$ of a dipole moving to the right and $(b)$ the interpolated velocity field for the case of a $30 \times 30$ grid. The spatial distributions of vorticity $\omega$ and stream function $\psi$ are derived from the interpolated velocity field and are shown in plots $(c)$ and $(d)$, respectively. The spatial distribution of the stream function $\psi^{\prime}$ relative to a comoving frame is shown in $(e)$, and contour plot $(f)$ represents the spatial distribution of Jacobian term $J(\omega, \psi)$. The measurement was taken at $t=90 \mathrm{~s}$ after injection; experimental parameters: $Q=12 \mathrm{ml} \mathrm{s}^{-1}, \delta t=0.3 \mathrm{~s}$ and $N=$ $2.2 \mathrm{rad} \mathrm{s}^{-1}$. 

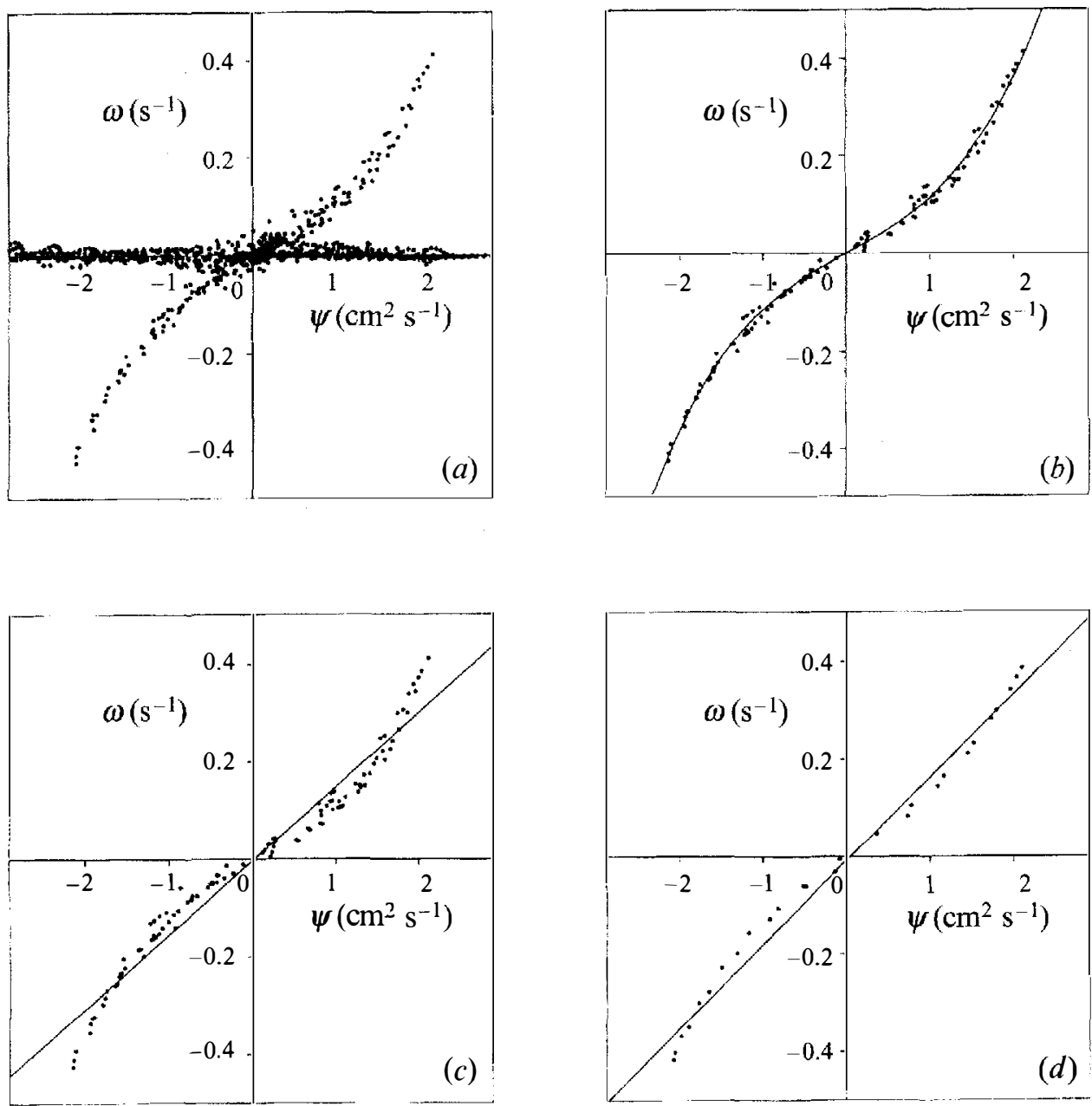

Figure 9. Typical $\left(\omega, \psi^{\prime}\right)$-scatter plot $(a)$ of the dipole of figure 8 obtained after correction for its translation $\left(U=22 \mathrm{~cm} \mathrm{~s}^{-1}\right)$. After zooming in on the actual dipolar structure, fits with the functions (b) $\omega=k_{n l}^{2} \psi+\beta \psi^{3}$ and $(c) \omega=k_{l i n}^{2} \psi$ were made by the least-square method. In graph $(d)$ a linear fit for the $\omega$ - and $\psi$-values along the cross-section through the vortex centres is shown.

times the dipole radius. At the grid edge the velocity between two neighbouring grid points was calculated directly from the cubic spline functions, and the stream function was determined with a central difference scheme. The validity of this procedure was tested by using the Lamb-Chaplygin dipole as a model flow field, with the solid flow boundaries at a distance comparable to that in the actual laboratory experiment: the characteristics of the interpolated velocity field were found to correspond quite well with those predicted by the theoretical model.

\subsubsection{The $(\omega, \psi)$-relationship}

An experimental representation of the functional relation (13) between the vorticity and the stream function for the present dipolar flow field can be obtained by plotting the value of $\omega$ against the value of $\psi^{\prime}$ (hereaf ter the prime is omitted again) for each of the 900 grid points in a so-called 'scatter plot'. For the experiment shown in figure 8 the corresponding scatter plot thus obtained is presented in figure $9(a)$. The points clustered in the horizontal band with $\omega=0$ all lie outside the dipolar vortex; and 
represent the exterior potential flow. The two branches with $\omega>0$ and $\omega<0$ each represent one half of the dipole. Although some scatter is observed, the points collapse quite well onto a single curve, whose shape resembles a sinh-like profile. This nonlinear relationship has been found in the majority of experiments in which the dipole emerged from the collapse of the turbulent patch. The scatter plot is obtained after correction for the dipole's translation, the speed of which was directly measured from the displacement of the virtual vortex centres in the subsequent streak photographs. A similar nonlinear $(\omega, \psi)$-relationship was found by Nguyen Duc \& Sommeria (1988) in their experiments on two-dimensional dipole vortices in mercury.

Although the vortex structure in the present experiments is three-dimensional, according to the scaling analysis of $\S 4.1$ the flow can to leading order be considered as quasi-two-dimensional, so that a comparison with a two-dimensional dipole model is a natural first step to take. The Lamb-Chaplygin model discussed in $\S 4.2$ provides such a reference. Even though the shape of the measured $(\omega, \psi)$-relationship is at variance with the linear proportionality (14) that forms the basis of this theoretical model, in view of the overall resemblance it is useful to make a further, more detailed, comparison between the experimental dipole structures and those of the Lamb-Chaplygin model.

\subsubsection{The translation speed}

As stated, the translation velocity of the laboratory dipole can be directly measured from the set of subsequent photographs. This actual translation speed $U_{\text {exp }}$ can be compared with the speed $U_{0}=\Gamma / 6.83 a$ predicted by the Lamb-Chaplygin model, see (17), with the circulation $\Gamma=\frac{1}{2}\left(\Gamma_{+}-\Gamma_{-}\right)$being obtained by integrating the positive and negative vorticity over all the grid points, and the radius $a$ being determined from the stream-function contour plot (see figure $8 e$ ). It is estimated that the value of the speed $U_{0}$ thus derived will be accurate to $6 \%$. In addition, by measuring the velocity $U_{\max }^{*}$ associated with the longest particle path on (or near) the axis of the dipole one obtains another estimate for the translation speed according to the Lamb-Chaplygin model, since $U_{0}^{*}=U_{\max }^{*} / 3.49$. Provided that the particular streak is not too distant from the dipole axis, this procedure is expected to be relatively accurate, with an estimated accuracy of approximately $4 \%$.

In an alternative approach the translation velocity can also be obtained indirectly from the scatter plots, i.e. by optimizing the fit of the points with some prescribed curve. A numerical routine was applied to minimize the standard deviation of the leastsquares fit of the $(\omega, \psi)$-points in the scatter plot to a third-order polynomial

$$
\omega=k_{n l}^{2} \psi+\beta \psi^{3}
$$

by iteratively varying the translation velocity $U_{\text {fit }}$. The result of such an optimal fit is shown graphically in figure $9(b)$, in which by focusing only the grid points in the dipole's interior were taken into account. It is clearly seen that the points collapse onto a single line, thus confirming that for that particular value of $U_{f i t}$ a quasi-stationary dipole structure is obtained (the minor scatter being attributed to the differentiation procedure applied to obtain the vorticity from the interpolated velocity field). The translation velocity $U_{f i t}$ is estimated to be accurate to within $6 \%$. The third-order polynomial (23) is an obvious choice to fit the antisymmetric $(\omega, \psi)$-data points. Moreover it approaches quite well the first two terms of the series expansion of the function $\omega=C \sinh \left(k^{2} \psi\right)$, which has been derived to apply under certain conditions to steady-state two-dimensional flow structures (see Joyce \& Montgomery 1973; Robert \& Sommeria 1991; Montgomery et al. 1992; Pasmanter 1994). For the experiment 


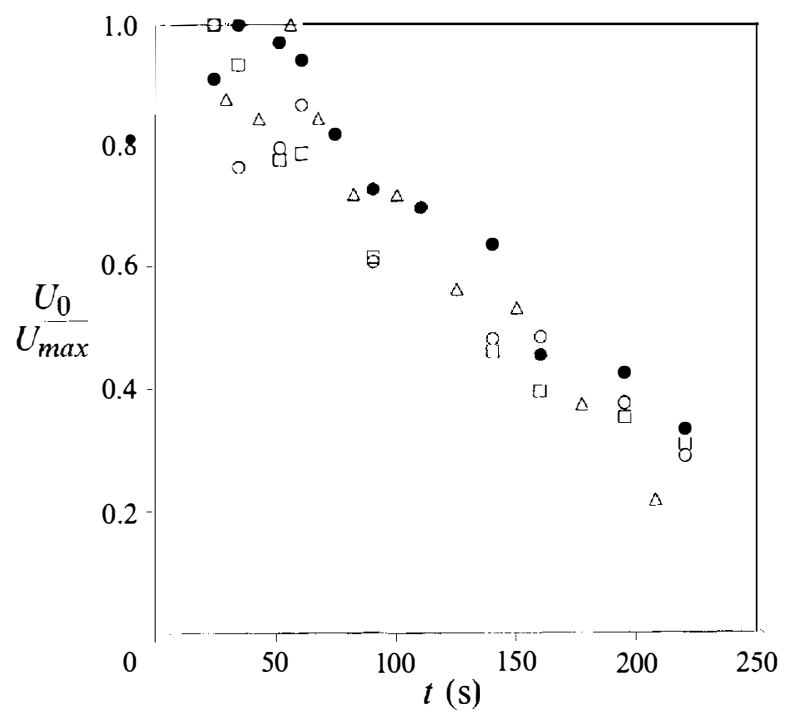

FIGURE 10. Graphical representation of the decaying translation velocity of the dipolar structure. The translation velocities were determined in different ways (see text): $\triangle, U_{\text {erp }} ; \square, U_{0} ; \boldsymbol{O}, U_{0}^{*} ; \bigcirc, U_{\text {fit }}$. Each one is scaled with its maximum value, which are $0.32,0.33,0.40$ and $0.36 \mathrm{~cm} \mathrm{~s}^{-1}$ for $U_{\text {exp }}, U_{0}$, $U_{0}^{*}$ and $U_{f i t}$, respectively. Experimental data: see figure 8.

shown in figure $9(b)$ these terms yield $C k^{2}=0.089 \pm 0.004$ and $C k^{6} / 6=0.015 \pm 0.002$, and correspond well with the values obtained by the optimal fit (23), namely $k_{n l}^{2}=$ $0.084 \pm 0.003$ and $\beta=0.020 \pm 0.03$, respectively. In the $k^{2} \psi$-range investigated here, higher-order terms of the sinh-function are small compared to the first two terms and could be neglected within the experimental error. It should be stressed here, however, that the sinh-relationship as derived in the above-mentioned theoretical studies is only obtained for specific initial distributions of vorticity. In $\S 6$ it will be shown that the measured $(\omega, \psi)$-relationship fits equally well to a relationship derived by Pasmanter (1994) for a different initial distribution.

The values of the translation velocities $U_{e x p}, U_{\bullet}, U_{0}^{*}$ and $U_{f i t}$ obtained during the evolution of the (gradually decaying) dipole are presented graphically in figure 10. It is observed that all data lie within a narrow band, thus revealing good agreement. Apparently, the Lamb-Chaplygin dipole model gives the correct estimate of the translation speed for the laboratory dipole with the nonlinear $(\omega, \psi)$-relationship.

As can be observed both from the dye visualizations (figure 5) and the contour plots of the corrected stream function (figure $8 e$ ), the dipole shape is to a good approximation circular, as in the Lamb-Chaplygin model. In addition, both from the dye visualizations and from the vorticity contour plots (e.g. figure $8 c$ ) the distance between the vortex centres is found to be $d / a=0.97 \pm 0.03$, which shows good agreement with the value $d / a=0.96$ according to the Lamb-Chaplygin model.

In view of the above results one might question whether the nonlinearity in the $(\omega, \psi)$-plot is just a small (local) perturbation of the linear $(\omega, \psi)$-relation. In that case, the size and the distance between the apparent vortex centres would be determined by the linear term in the third-order polynomial. In order to check this conjecture, the constant $k_{n l}$ appearing in the coefficient of the linear term of (23) is derived from the least-square fit of this third-order polynomial with the $(\omega, \psi)$-data (see figure $9 b)$. The distance between the virtual vortex centres could be measured - independently from any other data manipulation - from streak photographs. The values of $k_{n l} d_{v c}$ thus 


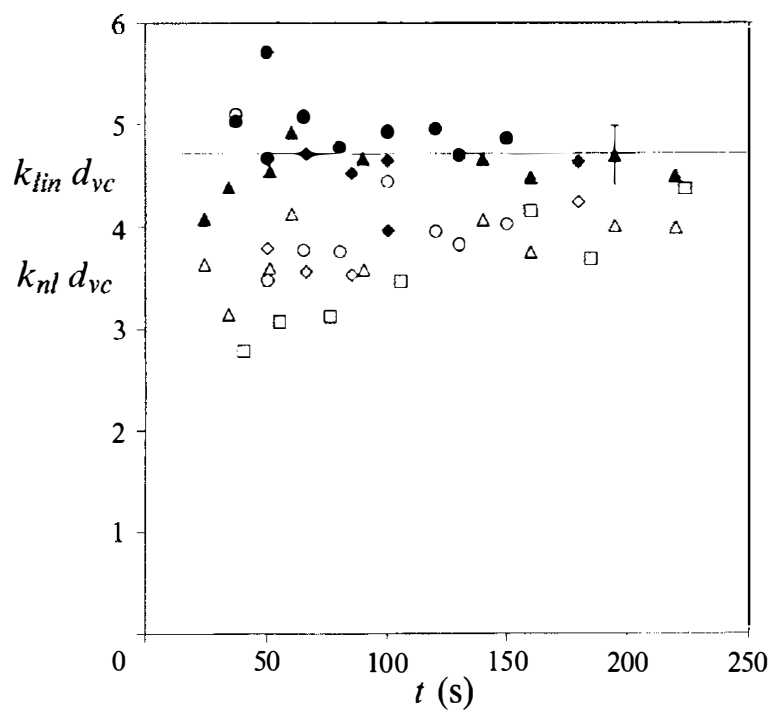

FIGURE 11. Graphical representation of the evolution of $k_{n i} d_{v c}$ (denoted by open symbols) and $k_{\text {tin }}$ $d_{v c}$ (denoted by filled symbols) against time. According to the Lamb-Chaplygin model $k d_{v e c}=4.72$. Experimental parameters: $\triangle, \boldsymbol{\Delta}$, see figure 8; $\bigcirc, \boldsymbol{\Theta}, Q=7.6 \mathrm{ml} \mathrm{s}{ }^{-1}, \delta t=0.8 \mathrm{~s}$ and $N=2.1 \mathrm{rad} \mathrm{s}^{-1}$; $\square, \square, Q=15.2 \mathrm{ml} \mathrm{s}^{-1}, \delta t=0.2 \mathrm{~s}$ and $N=2.1 \mathrm{rad} \mathrm{s}^{-1} ; \diamond, \diamond, Q=11.7 \mathrm{ml} \mathrm{s}^{-1}, \delta t=0.26 \mathrm{~s}$ and $N=2.3 \mathrm{rad} \mathrm{s}^{-1}$.

obtained are plotted in figure 11 (open symbols) against time for a sequence of streak pictures of different experiments and appear to have a mean value of $k_{n l} d_{v c}=3.8$, which is systematically lower than the value $k d_{v c}=4.72$ according to the LambChaplygin model. However, when the constant $k_{\text {lin }}$ is derived from a least-square fit $\omega=k_{l i n}^{2} \psi$ with the $(\omega, \psi)$-data as shown in figure $9(c)$, a mean value $k_{l i n} d_{v c}=4.6$ is obtained, which shows a much better agreement with the Lamb-Chaplygin model. Likewise, the dipole radius estimated from contour plots revealed good agreement with the radius derived by the relation $a=3.83 / k_{\text {lin }}$ for the Lamb-Chaplygin dipole. Although the nonlinearity in the $(\omega, \psi)$-plot suggests that strictly the Lamb-Chaplygin model does not apply, when averaged over the whole structure by assuming an overall linear $(\omega, \psi)$-relation (see figure $9 c$ ) fair agreement with the Lamb Chaplygin dipole is obtained. One might question now whether the nonlinearity possesses a spatial dependence, in the sense that the nonlinearity is mainly confined to for example the vortex centres and not to the boundaries, or whether it is evenly distributed over the whole structure. This will be addressed by investigating cross-sectional distributions of some relevant flow quantities.

\subsubsection{Cross-sectional distributions of velocity and vorticity}

The information available from the digitization of the flow field can also be used to plot the distribution of flow quantities such as the vorticity along certain cross-sections of the flow field. A convenient cross-section for the dipole flow is the line through its vortex centres, i.e $\theta= \pm \pi / 2$, along which the velocity is directed perpendicularly, i.e. $v(r, \pm \pi / 2)=\left(0, v_{\theta}(r, \pm \pi / 2)\right)$. Typical velocity and vorticity profiles along that cross-section are presented in figure $12(a, b)$. The values in between the gridlines were calculated by linear interpolation; the profiles were obtained by plotting the values of the velocity and vorticity as a function of $r$, the distance from the dipole centre. In order to compare the measured velocity distribution with that predicted by the Lamb-Chaplygin model, it is necessary to scale both the velocity and the radius. As 

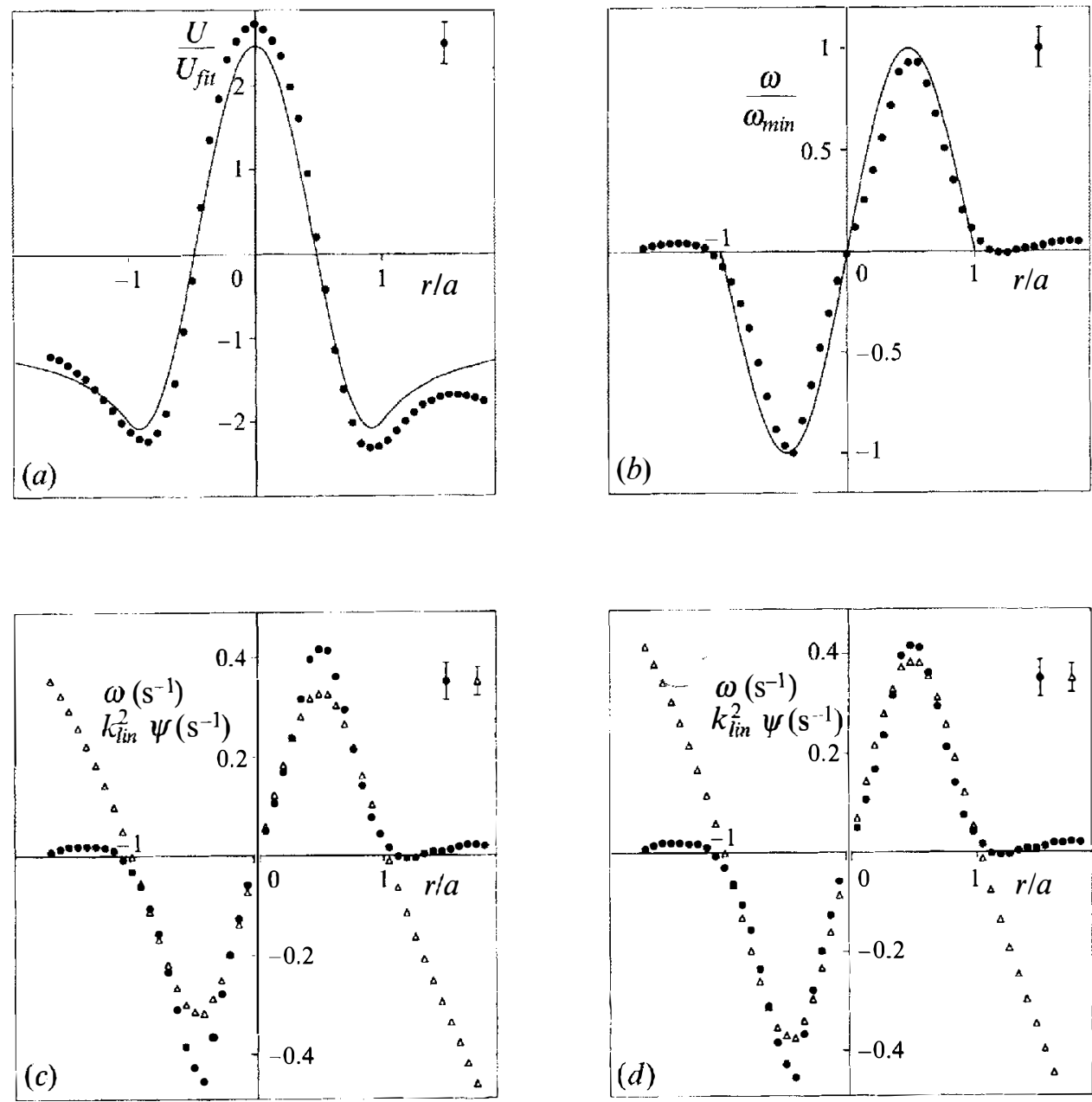

FIGURE 12. Characteristic cross-sectional distributions along a line through the vortex centres of the dipole shown in figure 8: $(a)$ the measured velocity profile $v_{\theta}$ (denoted by dots), scaled with $U_{f i t}=$ $0.22 \mathrm{~cm} \mathrm{~s}^{-1}$, relative to a comoving frame of reference; $(b)$ the derived vorticity (denoted by dots) scaled with the extreme value $\left|\omega_{m i n}\right|=0.49 \mathrm{~s}^{-1}$. Both experimental data sets are compared with the Lamb-Chaplygin model (solid curve). In (c) the values of $\omega$ and $k_{l i n}^{2} \psi$ are plotted along the same cross-section. The radial coordinate in $(a),(b)$ and $(c)$ is scaled with the dipole radius $a=3.831 / k_{l i n}=$ $9.8 \mathrm{~cm}$. In $(d) k_{l i n}$ is obtained from the least-square fit shown in figure $9(d)$.

before, we have used the translation speed $U_{f i t}$ as the velocity scale. The radial distance $r$ has been scaled with the radius $a$ according to the Lamb-Chaplygin dipole, for which $a=3.831 / k_{\text {lin }}$, where $k_{\text {lin }}$ is determined from a linear least-squares fit with the data in the $(a), r)$-scatter plot. The vorticity profile has been scaled by the extremal vorticity values. In both figures $12(a)$ and $12(b)$ the solid line represents the corresponding distribution according to the Lamb-Chaplygin model. The data points clearly show good agreement with the Lamb-Chaplygin curves, both inside the dipolar structure and in the exterior flow around it. The differences in the observed velocities at the dipole's symmetry axis $(r=0)$ and at its boundary $(r / a=1)$ as visible in figure $12(a)$ are attributed to the approximation of the translation speed. It turns out that fitting with $U_{0}^{*}$ leads to the best correspondence.

The comparison between the experimentally determined vorticity profile and that according to the Lamb-Chaplygin model (figure 12b) reveals that the experimental 
distribution is more 'peaked' near the vorticity extremes, although the positions of the extremal points again correspond with those of the Lamb-Chaplygin dipole. Also, the experimental points reveal the existence of regions of opposite vorticity just outside the main dipolar region, which are absent (by definition) in the Lamb-Chaplygin model. Furthermore, the experimentally found $\omega$-distribution has a smooth appearance near the edge $r / a=1$, whereas the corresponding Lamb-Chaplygin profile shows a kink there. These features have been observed in many other experiments, and are believed to be characteristic of the dipoles thus produced. Thus far, an explanation for the oppositely signed vorticity shield has not been found.

In order to gain some more insight in the spatial structure of the $\left(\omega, \psi^{\prime}\right)$-relationship, the values of $\omega$ and of $k_{l i n}^{2} \psi$ are plotted along the same cross-section, see figure 12(c), with $k_{\text {lin }}$ being determined from the linear fit of the $(\omega, \psi)$-data (see figure $\left.9 c\right)$ ). As shown above, when the radius $a$ is determined from the $\psi$-contour plot (figure $8 e$ ), the value $k_{l i n}^{2}$ thus obtained is in agreement with the dispersion relation (16). Grid points at which the values of $\omega$ and $k_{l i n \psi}^{2}$ are equal indicate a linear relationship. It is observed that the nonlinearity increases towards the vortex centres (i.e. with increasing value of $\psi)$ and has a maximum in the vortex centre itself. Apparently this structure is, in comparison with the Lamb-Chaplygin model, nonlinear, in particular in the vicinity of the vortex centres, while it is linear in the regions with low vorticity. When $k_{\text {lin }}^{2}$ is determined locally, for example by a linear least-square fit of the $\left(\omega, \psi^{\prime}\right)$-values over the present cross-section (see figure $9 d$ ), the nonlinearity is distributed over the whole dipolar structure, as is visible in figure $12(d)$. However, this $k_{\text {lin }}^{2}$ implies from (16) a different radius than measured from the $\psi$-contour plot and also yields a different value of $k_{l i n} d_{v c}$. Thus, the linear fit of the $(\omega, \psi)$-data obtained along this cross-section yields values of $k_{\text {lin }}$ and related quantities which are at variance with those according to the Lamb-Chaplygin model.

\subsection{Laminar-injection dipoles}

Thus far attention has been focused on dipolar vortices emerging after the gravitational collapse of a three-dimensional turbulent region generated by a pulsed turbulent injection of a small amount of fluid. It is known (see e.g. Voropayev et al. 1991) that laminar injections may give rise to dipolar vortices with a very similar appearance. Under such laminar conditions the size of the eventual dipole is determined by the total momentum of injected fluid. Therefore, relatively long injection times are required to obtain a dipole with a sufficiently large size and a sufficient long lifetime to analyse its flow characteristics. In order to make a comparison with the turbulent-injection dipoles discussed before, we have carried out a few experiments in which the dipole was created by laminar injection. In these experiments the Reynolds number based on the injection parameters (nozzle diameter, injection speed) was kept small, at a value of typically 450 .

It was observed that the front of the relatively narrow jet splits up into two elongated counter-rotating vortices, which are pushed forward by the injected fluid. For long injection times, the jet may show a meandering behaviour, with smaller vortices arranged on either side. Once the injection is stopped, the two frontal vortices get separated from the feeding stream and propagate forward in the form of a coherent dipolar vortex structure. Owing to the large concentration of linear momentum on the axis of this structure - a remnant of the forcing - the dipole is elongated in the axial direction, and also shows a pronounced head-tail asymmetry. During the course of the experiment, however, the dipole is observed to relax towards an approximately circular shape, with a very weak head-tail asymmetry. This evolution is clearly visible in the sequence of $(s-$ and $\psi$-contour plots presented in figure 13. A similar head-tail 


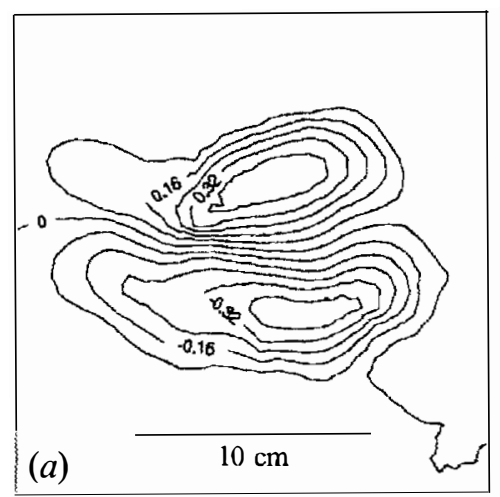

$\omega$

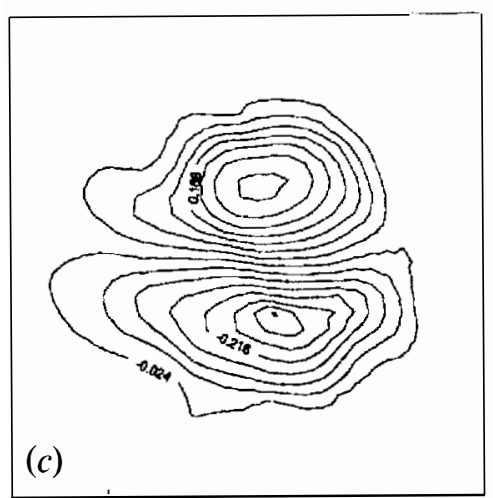

$\omega$

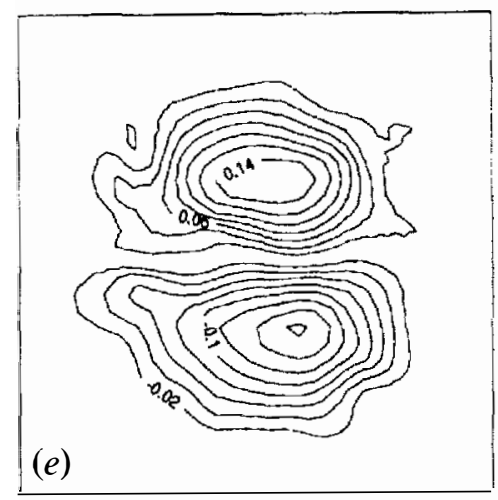

$\omega$

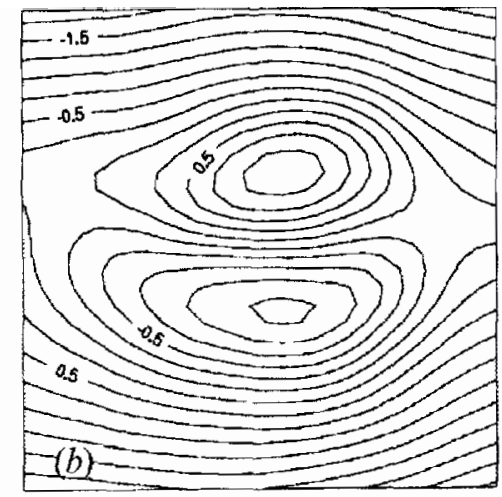

$\psi$

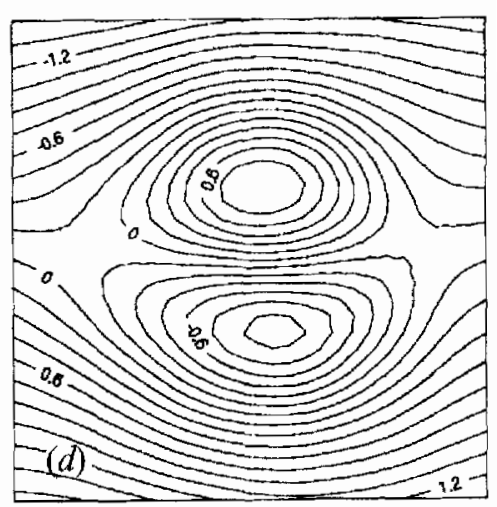

$\psi$

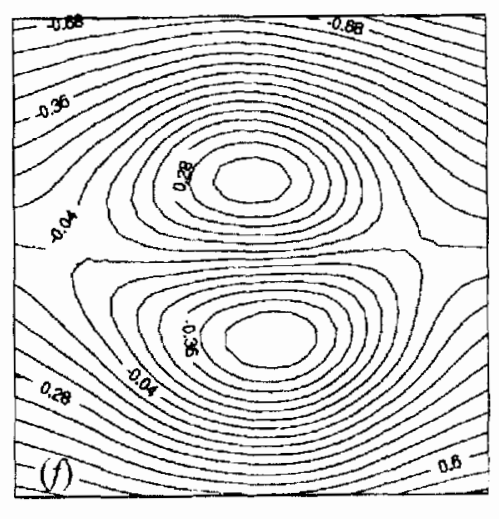

$\psi$

Figure 13. Contour plots of vorticity $\omega$ (left column) and corresponding stream function $\psi^{\prime} r^{\prime}$ (right column) for a dipole created by a laminar in jection of fluid. The dipole moves to the right. $(a, b) t=33 \mathrm{~s},(c, d) 64 \mathrm{~s}$ and $(e, f) 121 \mathrm{~s}$. Experimental parameters: $Q=0.58 \mathrm{ml} \mathrm{s}^{-1}, \delta t=25 \mathrm{~s}$ and $N=3.0 \mathrm{rad} \mathrm{s}^{-1}$.

asymmetry and an oval dipole shape was observed in a numerical study of dipolar vortices in a viscous flow with an external strain field, see Kida, Takoaka \& Hussain (1991). In the present experiments, however, the asymmetry and the elongated shape are not due to any external strain, but to the initial forcing with a jet. 


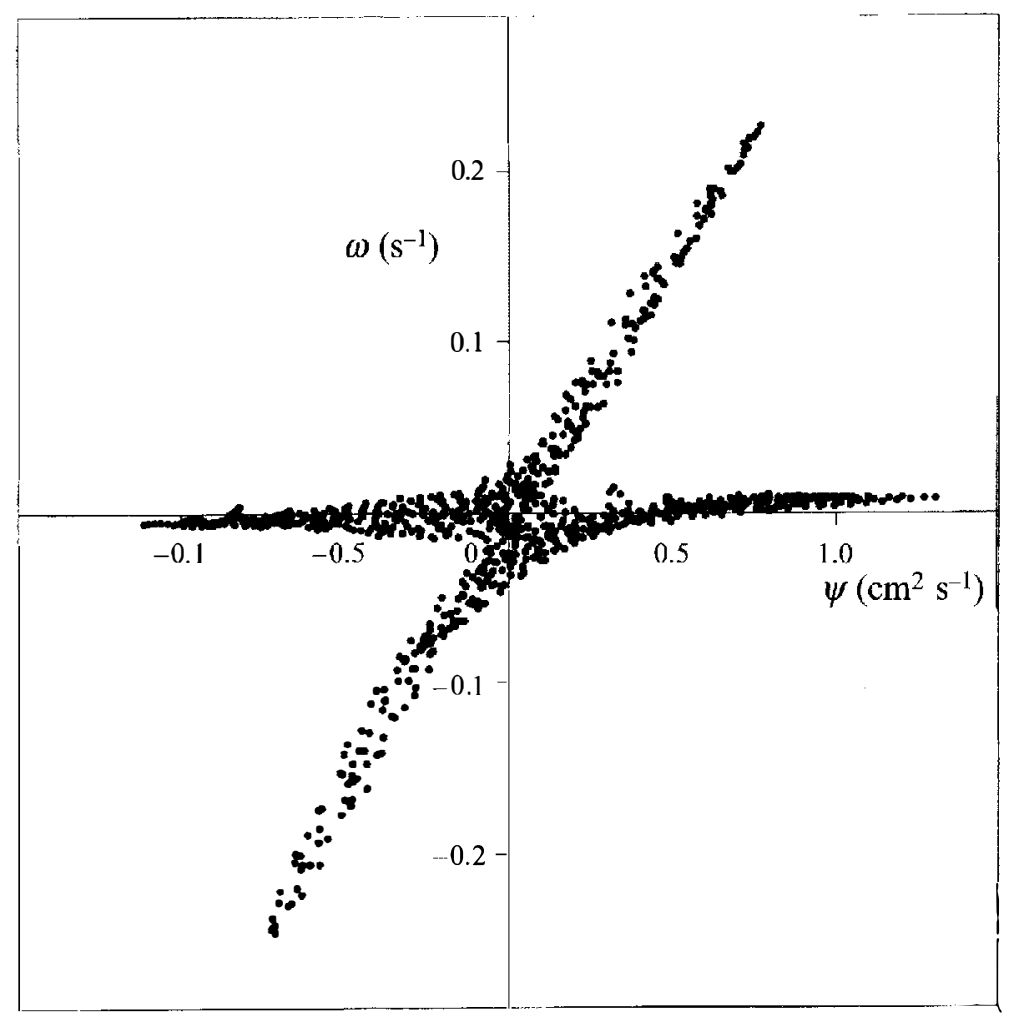

FIGURE 14. Characteristic $\left(\omega, \psi^{\prime}\right)$-scatter plot for the dipole created by laminar in jection of fluid.

The plot is taken from the experiment shown in figure 13 and is measured at $t=89 \mathrm{~s}$.

The $(\omega, \psi)$-scatter plot of the developed vortex dipole of the experiment shown in figure 13 is given in figure 14 . The points inside the dipole clearly define a straight line, implying a linear relationship $\omega=F(\psi)$ for this type of dipole. It was found that this linearity persisted throughout the dipole's further evolution. As remarked before, the dipole relaxes towards a circular shape, and the distance between the vortex centres gradually increases accordingly. In the developed stage and during the further evolution of the dipole, the value of $k d_{v c}=4.62$ corresponds very well with the value 4.72 according to the Lamb-Chaplygin model.

Concerning the dipole translation speed, the velocities $U_{f i t}, U_{e x p}, U_{0}$ and $U_{0}^{*}$ have been determined as in the turbulent-injection experiments ( $\$ 5.1 .3$ ), and again show a very good agreement throughout the experiment. This implies that the LambChaplygin model again applies very well for the description of this type of vortex dipoles. As before the comparison is carried further, by plotting the vorticity as well as the values of both $\omega$ and $k^{2} \psi$ along a cross-section through the vortex centres, see figure 15 . The measured vorticity profile (figure $15 a$ ) shows a very good correspondence with the Lamb-Chaplygin profile, the minor differences at the edge $r / a=1$ being attributed to viscous effects. The linearity of the $\omega=F(\psi r)$ relationship is reflected in the data points of $\omega(\mathrm{r})$ and $k^{2} \psi(r)$ shown in figure 15(b): the agreement (for $r / a<1$ ) is within the experimental error.

\subsection{Decay}

Once the vortex dipole is formed, it travels quasi-steadily along a straight trajectory. While travelling, however, the flow structure slowly decays by viscous effects, as can be seen from a decreasing internal velocity field and a decreasing translation speed. Visual 

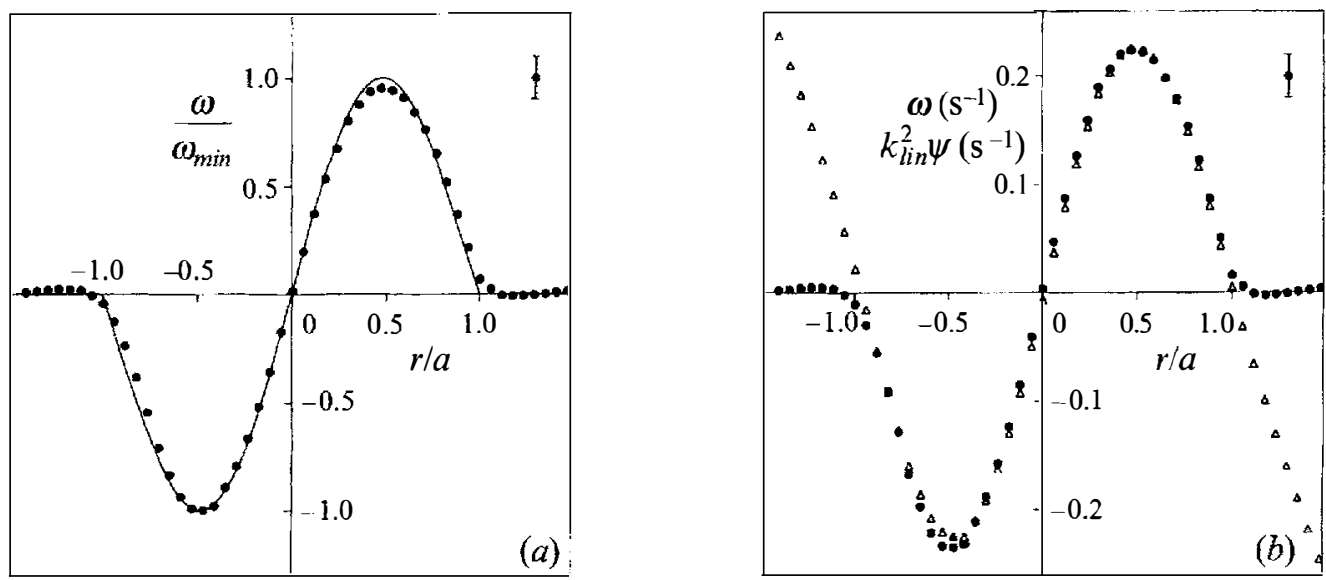

FIGURE 15. Characteristic cross-sectional distributions of flow properties of the dipole shown in figure 14 created by a laminar injection. As in figure 12 the cross-section is taken through the vortex centres. Graph $(a)$ presents the measured vorticity (dots) scaled with the extremal value $\left|\omega_{\min }\right|=0.25 \mathrm{~s}^{-1}$; the solid curve line depicts the (scaled) $\omega$-profile according to the Lamb-Chaplygin model. The values of $\omega$ and $k_{l i n}^{2} \psi$ along the same cross-section are shown in $(b)$. The radial coordinate in both plots is scaled with the radius $a=3.831 / k_{l i n}=6.9 \mathrm{~cm}$.

observations suggest a shape preservation, although the vortex gradually expands during the decay process. In order to investigate whether the dipole characteristics change during the decay, we have determined the $(\omega, \psi)$-scatter plots for a number of subsequent stages in the evolution, and for the turbulent-injection case the results are presented in figure 16. It is seen that, although the maximum vorticity amplitude and the average slope of the $(\omega, \psi)$-curve both gradually decrease, the shape of the $(\omega, \psi)$ curve is conserved. This property was tested more rigorously by fitting the subsequent curves with the third-order polynomial (23). For the scaled plots (normalized by the extreme values) the coefficients of this polynomial appeared to be virtually constant in time, illustrating a self-similarity of the $(\omega, \psi)$-relation during the decay. In the long run, however, figure 11 shows for a number of experiments that the (averaged) values $k_{n l}$ and $k_{l i n}$ converge to the same value, suggesting a tendency towards a linear $(\omega, \psi)-$ relation. Detailed information about the structure during its decay can be obtained from a sequence of $\omega$-cross-sections along the line through the vortex centres in time, as is displayed in figure $17(a)$ (for the turbulent-injection dipole with the nonlinear $\omega$, $\psi$-relation) and in figure $17(b)$ (for the laminar-injection dipole with the linear $\omega, \psi$ relation), respectively. The linking between positive and negative vorticity patches appears to be weak for the nonlinear case, as is apparent from the low-vorticity region between the vortex centres, while the linking is strong for the linear case, indicating that entrainment is less effective. The difference in linking is probably due to the difference in the generation process: the flattening of the vortical region by the collapse (which is absent for laminar injections) increases the separation between the vortex centres at the initial dipole formation, which leads to an increase of entrainment of ambient fluid. During the decay process this entrainment decreases and the differences between the linear and the nonlinear case become less pronounced. Figure 18 shows the values of the mean extremal vorticity $\frac{1}{2}\left(\omega_{\max }-\omega_{\min }\right)$ and the normalized mean vorticity $\Gamma / a^{2}$ as a function of time for the turbulent-injection dipole of figure 16, and the decay is obvious. The data have been fitted by straight lines, and the fair agreement with these lines suggests an exponential decay at approximately the same rate. As discussed in $\S 5.1$, the translation speed of the dipole decreases in an exponential fashion as well. 

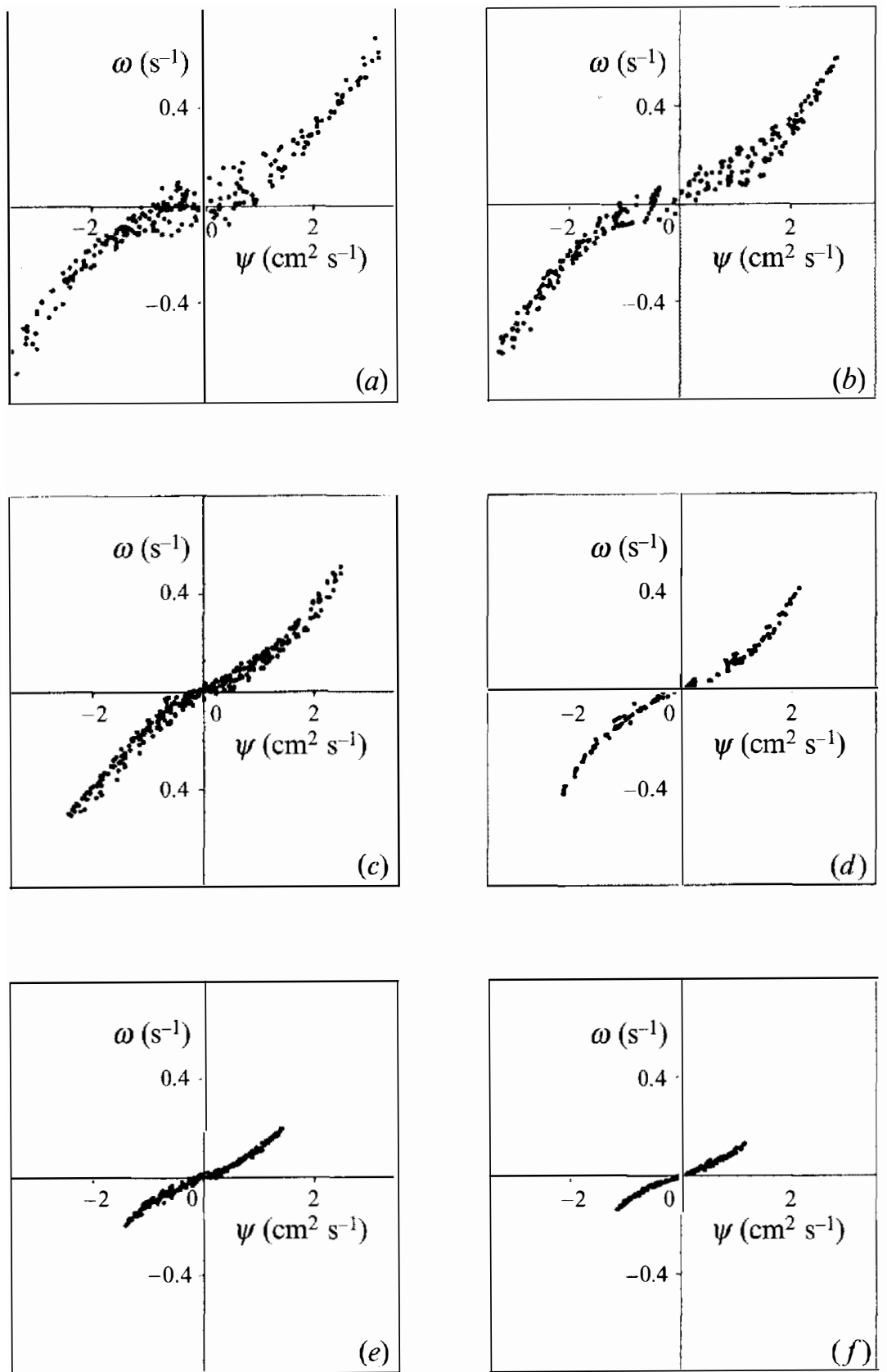

FIGURE 16. Sequence of $(\omega, \psi)$-scatter plots depicting the evolution in the $(\omega, \psi)$-relation during the decay process. For each plot a correction has been made for the translation velocity $U_{f i t}$; the surrounding points with zero vorticity have been eliminated by zooming in on the actual dipolar structure. (a) $t=34 \mathrm{~s},(b) 51 \mathrm{~s},(c) 60 \mathrm{~s},(d) 90 \mathrm{~s},(e) 160 \mathrm{~s}$ and $(f) 220 \mathrm{~s}$. Experimental parameters: see figure 8 .

In an analytical/numerical study, Swaters (1988) investigated the viscous evolution of a Lamb-Chaplygin dipole while allowing for horizontal diffusion of vorticity. $\mathrm{He}$ found that, while the dipole preserves its shape, the vorticity and the circulation as well as the translation speed decay exponentially. Although the present observations seem to correspond with this behaviour, in the case of a pancake-shaped vortex in a stratified 

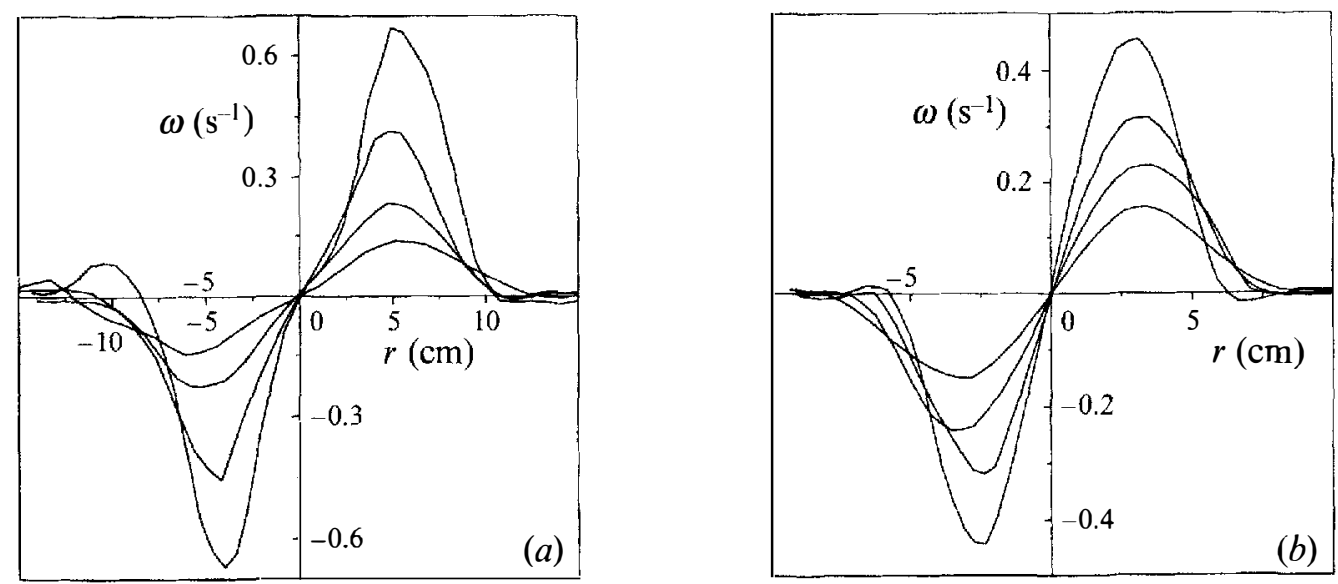

FIGURE 17. Graph showing the evolution of the vorticity profile along a cross-section through the vortex centres $(a)$ for the turbulent-injection dipole and $(b)$ for a laminar-injection dipole, characterized by a nonlinear and linear $(\omega, \psi)$-relation, respectively. The profiles of $(a)$ and $(b)$ are taken from the dipoles of figures 8 and 13, respectively. The profiles of $(a)$ were measured at $t=51 \mathrm{~s}$, $90 \mathrm{~s}, 140 \mathrm{~s}$ and $220 \mathrm{~s}$, and of $(b)$ at $t=30 \mathrm{~s}, 60 \mathrm{~s}, 85 \mathrm{~s}$ and $130 \mathrm{~s}$. Experimental parameters: $(a)$ see figure $8,(b)$ see figure 13 .

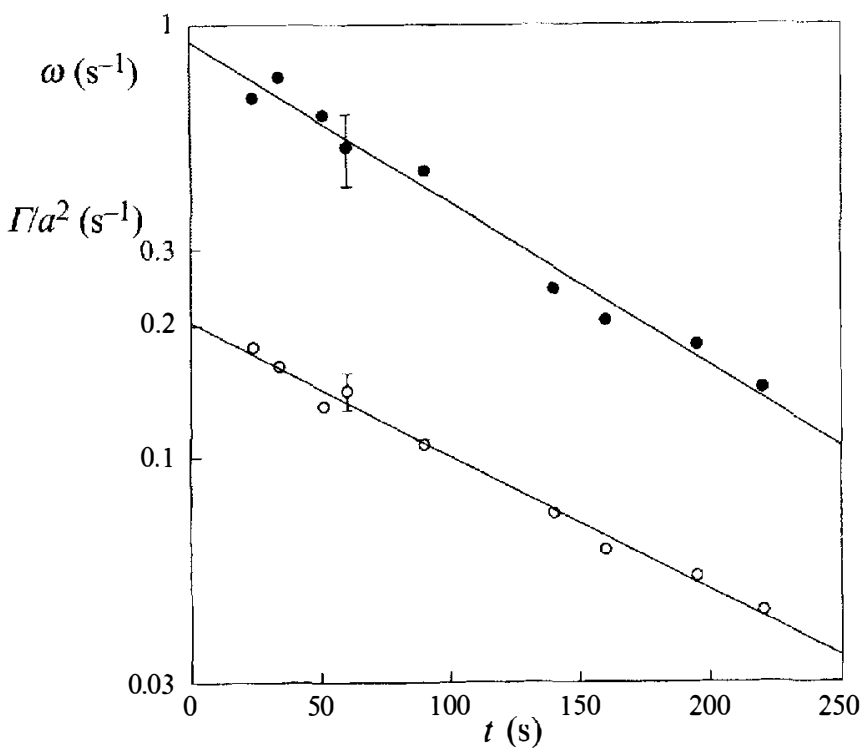

FIGURE 18. Graphical representation of the decay of the mean normalized circulation $\Gamma / a^{2}(O)$ and the mean extremal vorticity $\omega=\frac{1}{2}\left(\omega_{\max }-\omega_{\min }\right)(\mathbf{O})$. Both data sets are least-square fitted by exponential functions of the form $\mathrm{e}^{-\alpha t}$, with $\alpha$ a free parameter. Experimental parameters: see figure 8 .

fluid diffusion of vorticity occurs both in horizontal and in vertical directions, implying a much faster decay rate than in a purely two-dimensional flow. It has been found (see Flór, van Heijst \& Delfos 1994) that by including the effects of vertical diffusion a decay timescale is derived which agrees with the observations.

Continuous entrainment of ambient fluid at the rear causes a gradual expansion of the dipole, as can be seen in the increasing distance between the vortex centres, see figure 19. The data suggest that the size of the dipole increases linearly in time. 


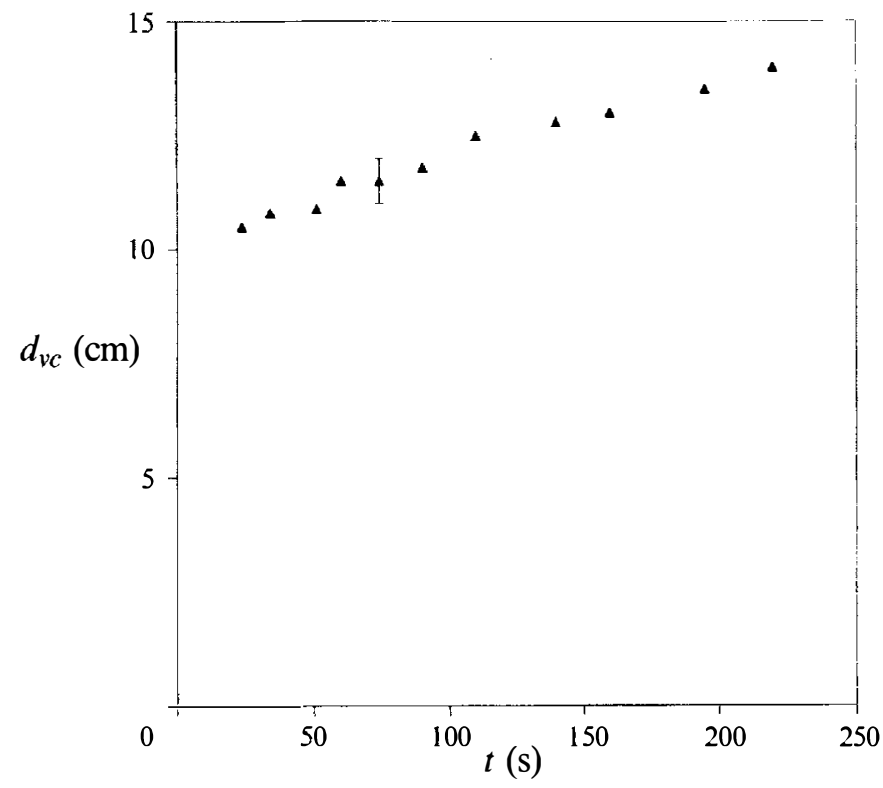

FIGURE 19. Graphical representation of the evolution of the distance between the virtual vortex centres $d_{x x}$ measured from streak pictures of the dipole experiment of figure 8 .

However, it was shown by Swaters (1988) that the radius of a viscously decaying Lamb-Chaplygin dipole remains constant under the (adiabatic) constraint that to leading order the dispersion relationship (16) and linear relation (14) are continuously maintained. In the present experiments, entrainment and-on large timescales viscous effects near the boundary cause the nonlinear term to become non-zero, so that the adiabatic assumption does not hold.

The entrainment of (irrotational!) ambient fluid at the rear of the dipole causes a head-tail asymmetry in the vortex structure. Since this is hard to observe from the isoline plots of $\omega$ and $\psi$, it was decided to plot the spatial distribution of the Jacobian term $J(\omega, \psi)$ in (12), which represents the advection of vorticity. In this approach it is assumed that changes caused by advection (entrainment) take place on a timescale that is small compared with the diffusion timescale. Figure $8(f)$ shows a contour plot of $J(\omega, \psi)$ for the uncorrected non-stationary flow field (i.e. without correction for the dipole translation) of figure $9(c, d)$. The spatial distribution has a characteristic quadrupolar structure, which is exactly what is to be expected for the case of a moving vortex dipole. Careful observation reveals, however, that $J(\omega, \psi)$ reaches larger values at the dipole's rear, which is consistent with the picture of the entrainment of ambient fluid occurring there.

\section{Discussion and conclusions}

The experiments described in this paper have shown that the dipolar vortices generated by pulsed horizontal injection of a small amount of fluid into a linearly stratified ambient fluid possess properties that are highly similar to those of purely twodimensional vortex dipoles. Although the dipoles in the stratified fluid occupy a characteristic flattened pancake-like volume, the associated flow field is planar. Measurements of the flow in the mid-plane have revealed that the vorticity is essentially distributed continuously, over an area that is close to circular. For this reason a 
comparison of the measured flow characteristics with the Lamb-Chaplygin model is an obvious step to take, and a fair agreement was found.

In some cases, in particular for the dipoles created by laminar injection, the $(\omega, \psi)$ relation was shown to be linear, as is also assumed in the Lamb-Chaplygin dipole model. Similar linear relationships were found in the MHD experiments by Nguyen Duc \& Sommeria (1988) and in the rotating-fluid experiments by Velasco Fuentes \& van Heijst (1994), both for the $f$-plane and for the topographic $\beta$-plane. In contrast, the dipoles in a stratified fluid generated by turbulent injection are characterized by a nonlinear relationship, which is close to $\omega=C \sinh k^{2} \psi$, approached in the present experiments by the cubic polynomial $\omega=k_{n l}^{2} \psi+\beta \psi^{3}$. In their statistical-mechanics approach of characterizing steady-state structures in two-dimensional flows, based on searching for the most probable state, Joyce \& Montgomery (1973) found evidence for the validity of this sinh-relationship for such two-dimensional flow structures. Some years later, numerical simulations by Matthaeus et al. (1991 $a, b)$ and Montgomery et al. (1992) revealed that organized two-dimensional flows obey the same relationship. Similar evidence was obtained by Robert \& Sommeria (1991) by maximum-entropy arguments, in particular for the case of 'dilute' vorticity distributions. The sinhrelationship strictly only applies to organized flows that originate from an initial state in which the vorticity has discrete values $\omega_{0}, 0$ and $-\omega_{0}$, with the total vorticity being zero. In a recent theoretical study, Pasmanter (1994) derived that for an initially Poisson-type vorticity distribution, the organized flow is characterized by an $\left(\omega, \psi^{\prime} r\right)$ relationship of the form

$$
\omega=-A \frac{\psi}{B^{2}-\psi^{2}},
$$

where $A$ and $B$ are constants whose values depend on the initial state. Figure 20 shows a comparison of the measured $(\omega, \psi)$-scatter plot both with the sinh-relationship and with (24). The theoretical curves are hardly distinguishable in the interval of the measured data, and seem to fit the measurements equally well. Obviously, the present experiments are not conclusive in determining which of the two theoretical relationships applies to organized dipolar flow.

In some cases, Nguyen Duc \& Sommeria (1988) observed dipolar vortices that are also characterized by a similar nonlinear $\left(\omega, \psi^{\prime}\right)$-relationship. The same type of relationship was found by Couder \& Basdevant (1986) in a numerical simulation of a vortex dipole resulting from two oppositely signed Gaussian monopolar vortices that were initially put close to each other. These authors pointed out that for this nonlinear case the dipole's constituent vortices showed a relatively weak linking, as can be observed in the vorticity distribution along a cross-section through the dipole centres, see e.g. figure 17 : the vorticity profiles suggest that the dipole halves are slightly shifted apart, with a region of low vorticity in between them. Most likely such a separation is associated with the entrainment of ambient fluid with zero vorticity at the rear of the dipole. This notion is once more stressed by the facts that during the decay this lowvorticity region disappeared while the global parameter $k_{n l} d_{v c}$ showed a tendency towards the value for a linear $(\omega, \psi)$-relation, and that for the dipoles with a linear $(\omega, \psi)$-relation a strong linking was observed.

In addition, it can be expected that the density distribution inside the dipolar vortex region has some effect. It could well be that a correction for the vertical density structure, obtained by plotting the potential vorticity rather than the vorticity $\omega$, as a function of $\psi$, yields a relationship that is less nonlinear than those presented in $\S 5$. However, since detailed measurements of the density distribution inside the dipole 


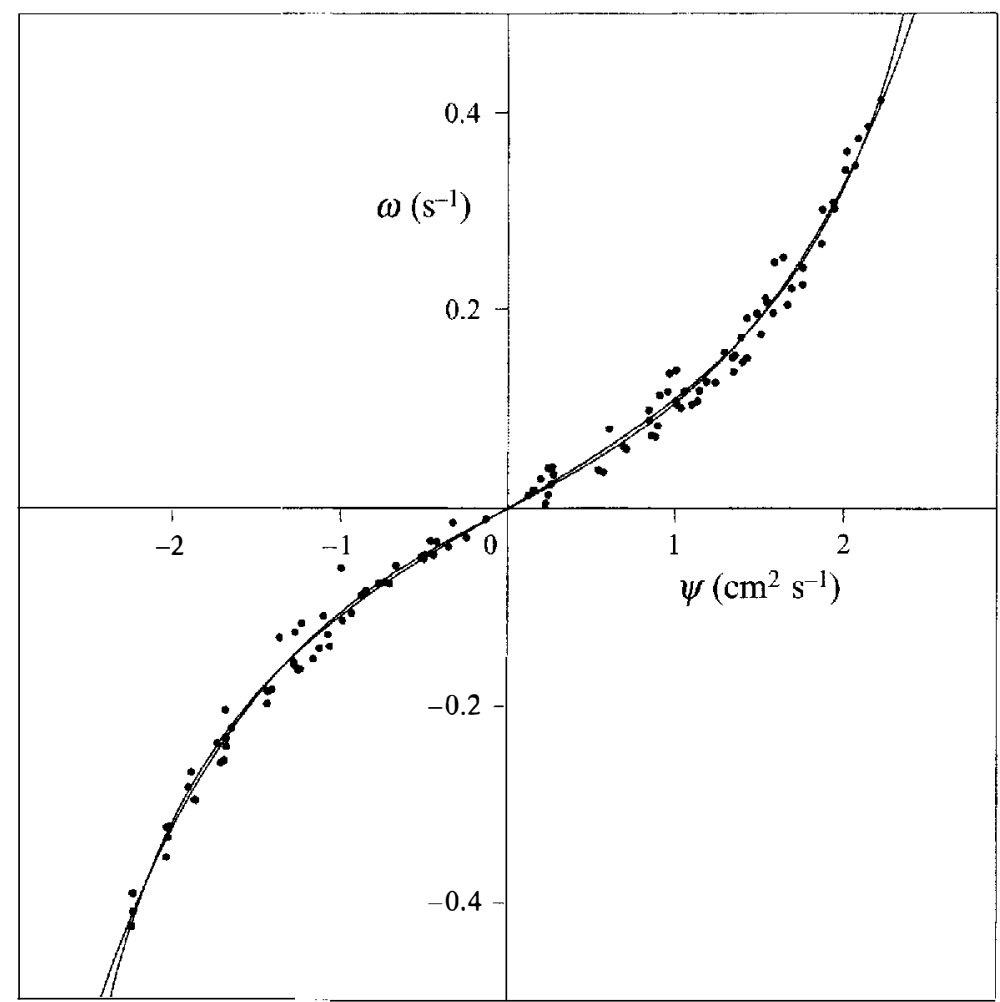

FIgURE 20. Typical $(\omega, \psi r)$-scatter plot fitted with the functions $\left.\omega=C \sinh \left(k^{2}\right\}\right)$ and $\psi=A \psi / r /$ $\left(B^{2}-\psi^{2}\right)$ by the least-square method. Experimental parameters: see figure 8. Data taken at $t=90 \mathrm{~s}$.

volume are at present not available, such a correction cannot yet be made. Notwithstanding the nonlinear character of the $(\omega, \psi)$-relationship, both the turbulentinjection and the laminar-injection dipoles show very good agreement with the theoretical Lamb-Chaplygin model, as is evident from the circular size, the crosssectional distributions of velocity and vorticity, the positions of the vorticity extremes and the translation speed of the dipole.

The authors thank Caspar Williams and Piet Jonker for their contributions to the further development of the image analysis system, which is partially based on sof tware kindly provided by Dr Joël Sommeria (Lyon, France), and to Professor David C. Montgomery and Dr Rubén A. Pasmanter for some helpful discussions. J.B.F. gratefully acknowledges financial support by the Foundation for Fundamental Research on Matter (FOM) of the Netherlands Organization for Pure Research (NWO).

\section{REFERENCES}

Batchelor, G. K. 1967 An Introduction to Fluid Dynamics. Cambridge University Press.

CAPERAN, P. \& Verron, J. 1988 Numerical simulation of a physical experiment on two-dimensional vortex merging. Fluid Dyn. Res. 3, 87-92.

Couder, Y. \& Basdevant, C. 1986 Experimental and numerical study of vortex couples in twodimensional flows. J. Fluid Mech. 173, 225-251.

Fernando, H. J. S., Heisst, G. J. F. van \& Fonshka, S. V. 1994 The evolution of an isolated turbulent region in a stratified fluid. J. Fluid Mech. (Submitted). 
Flór, J. B., Heisst, G. J. F. van \& Delfos, R. 1994 Decay of dipolar vortex structures in a stratified fluid. Phys. Fluids (in press).

Fortuin, J. M. H. 1960 Theory and application of two supplementary methods of constructing density gradient columns. J. Polymer. Sci. 44, 505-515.

GiBson, C. H. 1980 Fossil turbulence, salinity, and vorticity turbulence in the ocean. In Marine Turbulence (ed. J. C. J. Nihoul), pp. 221-257. Elsevier.

Heijst, G. J. F. van \& Flór, J. B. $1989 a$ Dipole formation and collisions in a stratified fluid. Nature $340,212-215$.

HeiJST, G. J. F. VAN \& FI.ÓR, J. B. $1989 b$ Laboratory experiments on dipole structures in a stratified fluid. In Mesoscale/Synoptic C'oherent Structures in Geophysical Turbulence (ed. J. C. J. Nihoul \& B. M. Jamart), pp. 609-626. Elsevier.

Honji, H., Tanfda, S. \& Tatsuno, M. 1980 Some practical details of the electrolytic precipitation method of flow visualization. Rep. Res. Inst. Appl. Mech. (Japan) 28, 83-89.

Hopfinger, E. J. \& Heust, G J. F. van 1993 Vortices in rotating fluids. Ann. Rev. Fluid Mech. 25, 241-289.

Joyce, G. \& Montgomiry, D. C. 1973 Statistical mechanics of negative temperature states. Phys. Fluids 17, 1139-1145.

Kida, S., Takoaka, M. \& Hussain, F. 1991 Formation of head-tail structure in a two-dimensional uniform straining flow. Phys. Fluids A 3, 2688-2697.

LamB, H. 1932 Hydrodynamics (6th edn.) Cambridge University Press.

LILLY, D. K. 1983 Stratified turbulence and the mesoscale variability of the atmospherc. J. Atmos. Sci. 40, 749-761.

Lin, J. T. \& PaO, Y. H. 1979 Wakes in stratified fluids. Ann. Rev. Fluid Mech. 11, 317-338.

Matthaeus, W. H., Stribling, W. T., Martinez, D., Oughten, S. \& Montgomery, D. C. 1991 a Decaying, two-dimensional, Navier-Stokes turbulence at very long times. Physica D 51, 531-538.

Matthaeus, W. H., Stribling, W. T., Martinez, D., Oughton, S. \& Montgomery, D. C. $1991 b$ Selective decay and coherent vortices in two-dimensional incompressible turbulence. Phys. Rev. Lett. 66, 2731-2734.

Meleshoo, V. V. \& Heisst, G. J. F. van 1994 On Chaplygin's investigations of two-dimensional vortex structures in an inviscid fluid. J. Fluid Mech. 272, 157-182.

Montgomery, D. C., Matthaeus, W. H., Stribling, W. T., Martinez, D. \& Oughton, S. 1992 Relaxation in two-dimensions and the "sinh-Poisson" equation. Phys. Fluids A 4, 3-6.

Nguyen Duc, J. M. \& Sommeria, J. 1988 Experimental characterization of steady two-dimensional vortex couples. J. Fluid Mech. 192, 175-192.

Pasmanter, R. A. 1994 On long lived vortices in 2-D viscous flows, most probable states of inviscid 2-D flows and a soliton equation. Phys. Fluids 6, 1236-1241.

Pierrehumbert, R. T. 1980 A family of steady, translating vortex pairs with distributed vorticity. J. Fluid Mech. 99, 129-144.

Riley, J. J., Metcalfe, R.W. \& Whissman, M. A. 1981 Direct numerical simulations of homogeneous turbulence in density stratified fluids. In Proc. AIP Conf. Nonlinear Properties of Internal Waves (ed. B. J. West), pp. 79-112.

Robert, R. \& SOMmeria, J. 1991 Statistical equilibrium states for two-dimensional flows. J. Fluid Mech. 229, 291--310.

Swaters, G. E. 1988 Viscous modulation of the Lamb dipole vortex. Phys. Fluids 31, 2745-2747.

Velasco Fuentes, O. U. \& Heisst, G. J. F. van 1994 Experimental study of dipolar vortices on a topographic $\beta$-plane. J. Fluid Mech. 259, 79-106.

Voropayev, S. I. \& Filippov, I. A. 1985 Development of a horizontal jet in homogeneous and stratified fluids: Laboratory experiments. Izv. Akad. Nauk SSSR, Fiz. Atmos. Okeana 21, 964-972 (in Russian).

Voropayev, S. I., Afanasyev, Y. D. \& Filipyov, I. A. 1991 Horizontal jets and vortex dipoles in a stratilied fluid. J. Fluid Mech. 227, 543-566.

Wu, J. 1969 Mixed region collapse with internal wave generation in a density stratified medium. $J$. Fluid Mech. 35, 531-544. 